\title{
A Survey on Contact Distributions
}

\author{
Daniel Hug ${ }^{1}$, Günter Last ${ }^{2}$, and Wolfgang Weil ${ }^{3}$ \\ 1 Mathematisches Institut, Universität Freiburg, Eckerstr. 1, D-79104 Freiburg, Germany, \\ e-mail: daniel.hug@math.uni-freiburg.de \\ 2 Institut für Mathematische Stochastik, Universität Karlsruhe (TH), Englerstr. 2, D-76128 \\ Karlsruhe, \\ e-mail: g.last@math.uni-karlsruhe.de \\ ${ }^{3}$ Mathematisches Institut II, Universität Karlsruhe (TH), Englerstr. 2, \\ D-76128 Karlsruhe, \\ e-mail: weil@math.uni-karlsruhe.de
}

\begin{abstract}
This survey describes contact distributions of random structures from a geometric point of view. Various extensions of the classical contact distribution functions are considered. As a rule, we explain all concepts first in a simple situation, under the assumption of stationarity, and for Poisson point processes. In a second step we proceed to more complicated models. Particular emphasis is given to random patterns involving clustering.
\end{abstract}

\section{Introduction}

Contact distributions are tools to describe distributional properties of random spatial structures from outside the structure. For simplicity and in order to give a unified presentation, we consider random structures in a space $\mathbb{R}^{d}$ of general dimension $d \geq 2$. (Most results will remain true for $d=1$ if interpreted properly). The two types of random structures which we discuss are simple point processes $\Phi$ and random closed sets $Z$, where the second concept can be considered as a generalization of the first.

Suppose that $B \subset \mathbb{R}^{d}$ is a compact set which contains the origin 0 (we call $B$ a structuring element) and put $B^{*}:=-B$. For a reference point $x \in \mathbb{R}^{d}$ and a random set $Z$, we consider the (random) number $\rho \geq 0$ for which the first contact of the "growing" set $\rho B+x$ with $Z$ occurs. We can also adopt a slightly different point of view and define $\rho$ to be the random time it takes the growing set $Z+t B^{*}$ to reach $x$. The distribution of $\rho$ is a typical example of a contact distribution which is based on (generalized) distance measurements. If $Z$ is stationary, the contact distribution is independent of $x$, we then can choose $x=0$. If $Z$ has interior points, then typically (for example in the stationary case) $x \in Z$ with positive probability. In this situation, we will mostly consider the conditional distribution of $\rho$, given that $x \notin Z$. If $B$ is the unit ball, $\rho$ gives the Euclidean distance of $x$ to $Z$. For general structuring elements $B$, we therefore interpret $\rho$ as a $B$-distance. Besides the distribution of the $B$-distance, various other contact information can be taken into account, e.g. the direction in which the distance is attained or local geometric properties of $Z$ at the point of contact.

We will study these different types of contact distributions for random sets $Z$ and point processes $\Phi$. For many applications it is justified to assume that these random structures are stationary/spatially homogeneous (i.e. their distribution is translation invariant) or even stationary and isotropic, at least when considered on a suitable scale, 
e.g. as in cosmological models (see the contribution by C. Beisbart, M. Kerscher and K. Mecke, in Chap. 4 of this volume), or in appropriate subregions where the influence of the boundary can be neglected. For this reason, and since stationary models are easier to treat, we start with and for large parts concentrate on stationary structures. The formulae which we present also simplify for special classes of random sets resp. point processes. We therefore begin with Poisson processes in $\mathbb{R}^{d}$, then study particle processes and Boolean models, continue with cluster models and then treat the case of more general random sets. Some applications of contact distributions in the porous media literature are mentioned in [11. 26] (see also Chap. 1 'Shape of solids: porous and composite materials', in this volume), classical applications to forestry are reported in [43].

Extensions of results for contact distributions to not necessarily stationary models are more difficult to handle, since they usually involve mixed functionals [50] (compare also [26]). Therefore these generalizations will be discussed in a second step after the stationary case has been described carefully. It is evident that such extensions are required for the statistical analysis and modelling of inhomogeneous media [33]. Such inhomogeneous structures arise for instance if effects of external magnetic or gravitational fields cannot be neglected (compare [26]) or in materials formed by layers with a structural gradient near to their boundaries (gradient structures; see [5]).

The selection of the material for this tutorial paper was strongly influenced by our attempt to explain ideas related to our recent research and by trying to avoid too much overlap with existing introductions to the subject.

\section{$2 B$-Distances and Related Notions}

\section{$2.1 \quad B$-Distances}

Let $\mathcal{C}^{d}$ denote the class of all compact sets in $\mathbb{R}^{d}$. We fix a structuring element $B \in \mathcal{C}^{d}$ with $0 \in B$ and define the $B$-distance (the distance relative to $B$ ) of a point $x \in \mathbb{R}^{d}$ to the set $A \subset \mathbb{R}^{d}$ by

$$
d_{B}(x, A):=\inf \{r \geq 0:(x+r B) \cap A \neq \emptyset\} .
$$

The set on the right side may be empty (e.g. if 0 is a boundary point of $B$ or $A=\emptyset$ ), in which case $d_{B}(x, A)=\infty$. The distance $d_{B}(x, A)$ is translation invariant in the sense that $d_{B}(x+z, A+z)=d_{B}(x, A)$ for all $z \in \mathbb{R}^{d}$. Clearly, $d_{B}(x, A) \leq r$ if and only if $x$ is contained in the generalized outer parallel set $A+r B^{*}$ of $A$. Here, $C+D:=\{x+y: x \in C, y \in D\}$ is the (Minkowski) sum of two sets $C, D \subset \mathbb{R}^{d}$. For convenience we abbreviate $d_{B}(x, y):=d_{B}(x,\{y\})$, then

$$
d_{B}(x, y)=d_{B^{*}}(y, x), \quad x, y \in \mathbb{R}^{d} .
$$

We usually assume in the following that $B$ is a compact convex set with $0 \in B$. In this case we refer to $B$ as a gauge body. If $B$ is, in addition, full dimensional and centrally symmetric (w.r.t. 0$)$, then $(x, y) \mapsto d_{B}(x, y)$ is a metric on $\mathbb{R}^{d}$ induced by the norm $x \mapsto d_{B}(x, 0)$, and the pair $\left(\mathbb{R}^{d}, d_{B}\right)$ is called a Minkowski space. The choice $B=B^{d}$ (the Euclidean unit ball) yields the usual Euclidean metric which is based on the scalar product $\langle\cdot, \cdot\rangle$; we then write $d(x, A):=d_{B^{d}}(x, A)$. 


\subsection{Contact Vectors}

If the $B$-distance $d_{B}(x, A)$ of a point $x \notin A$ is attained in a unique point $y$ in the boundary $\partial A$ of $A$ (that means, if $\left.\left(x+d_{B}(x, A) B\right) \cap A=\{y\}\right)$, then we define the contact direction vector $u_{B}(x, A)$ as the element of $\partial B$ given by

$$
u_{B}(x, A):=\frac{y-x}{d_{B}(x, A)} .
$$

The points $x \in \mathbb{R}^{d} \backslash A$ for which the distance $d_{B}(x, A)$ is attained in more than one point of $A$ (and for which $u_{B}(x, A)$ is therefore not defined) form the exoskeleton $\operatorname{exo}_{B}(A)$ of $A$ (see [15] and also [40, 41] ). In the Euclidean case, and if $A$ is a finite or locally finite set, $\operatorname{exo}_{B^{d}}(A)$ is (the boundary of) the Voronoi tessellation generated by $A$. Subsequently, we write $V_{d}(A)$ for the Lebesgue measure of a Borel set $A \subset \mathbb{R}^{d}$. Then, if $A$ is a closed subset of $\mathbb{R}^{d}$ and $B \subset \mathbb{R}^{d}$ is compact, strictly convex and has 0 as an interior point, we have $V_{d}\left(\operatorname{exo}_{B}(A)\right)=0($ see [12, 14] $)$.

\section{Spatial Random Structures}

In this section, we give a short introduction to random sets and (different types of) point processes.

\subsection{Point Processes}

Point processes are not only fundamental objects of modern probability theory, but also provide basic models in applied probability; see [4] for a detailed exposition of the interesting and rich theory of point processes. Loosely speaking, a (simple) point process $\Phi$ on $\mathbb{R}^{d}$ is a finite or countable collection $\xi_{1}, \xi_{2}, \ldots$ of (mutually different) random points scattered in the $d$-dimensional Euclidean space $\mathbb{R}^{d}$. Each random variable $\xi_{n}$ is called a point of $\Phi$. We assume that the points of $\Phi$ do not accumulate in bounded sets, i.e. each bounded subset of $\mathbb{R}^{d}$ contains only a finite number of the points of $\Phi$. The points then form a locally finite closed subset $\left\{\xi_{1}, \xi_{2}, \ldots\right\}$ of $\mathbb{R}^{d}$.

To give a more formal definition of a point process, we introduce the space $\mathbf{N}$ of all locally finite sets $\varphi=\left\{x_{1}, x_{2}, \ldots\right\} \subset \mathbb{R}^{d}$. It is convenient to interpret a set $\varphi \in \mathbf{N}$ also as a counting measure, namely as $\varphi=\sum_{x} \delta_{x}$, where $\delta_{x}$ denotes the Dirac measure located at $x \in \mathbb{R}^{d}$ and where the sum extends over all $x$ which belong to $\varphi$. For simplicity, we write $\varphi=\sum_{n=1}^{\infty} \delta_{x_{n}}$, in the following, even if the sum is a finite one. Such a measure $\varphi$ is simple, that is each point has at most mass 1 , and locally finite, that is the number $\varphi(A)$ of points of $\varphi$ in $A \subset \mathbb{R}^{d}$ is finite for all bounded Borel sets $A$. A point process $\Phi$ is now a random element in $\mathbf{N}$ governed by an underlying probability measure $\mathbb{P}$. (To be mathematically more precise, one assumes some abstract probability space $(\Omega, \mathcal{A}, \mathbb{P})$ in the background and supplies $\mathbf{N}$ with the $\sigma$-field $\mathcal{N}$ generated by the mappings $\varphi \mapsto \varphi(A)$, where $A$ varies through all Borel sets in $\mathbb{R}^{d}$. Then, $\Phi$ is a measurable mapping from $\Omega$ to $\mathbf{N}$ and we assume hereafter that all random variables are defined on this probability space.) According to our conventions we regard $\Phi$ as a locally 
finite random closed set and, at the same time, as a simple random counting measure. Then $\Phi(A)$ is equal to the number of points of $\Phi$ that are in the set $A$, and $\mathbb{P}(\Phi(A)=n)$ is the probability that there are exactly $n$ points of $\Phi$ in $A$.

The intensity measure $\Lambda$ of $\Phi$ is the measure on $\mathbb{R}^{d}$ defined by

$$
\Lambda(A):=\mathbb{E} \Phi(A)=\sum_{n=1}^{\infty} n \mathbb{P}(\Phi(A)=n),
$$

where $A \subset \mathbb{R}^{d}$ is a Borel set and the symbol $\mathbb{E}$ denotes expectation with respect to the underlying probability measure $\mathbb{P}$, i.e. $\mathbb{E} \Phi(A)=\langle\Phi(A)\rangle$ in the notation used by physicists. The point process $\Phi$ is said to be stationary, if its distribution is invariant under translations, which means that $\mathbb{P}(\Phi+x \in C)=\mathbb{P}(\Phi \in C)$, for all $x \in \mathbb{R}^{d}$ and all events $C \in \mathcal{N}$. In this case, $\mathbb{P}(x \in \Phi)$ does not depend on $x \in \mathbb{R}^{d}$, so that Fubini's theorem implies

$$
\mathbb{P}(0 \in \Phi)=\int_{[0,1]^{d}} \mathbb{P}(x \in \Phi) d x=\mathbb{E} V_{d}\left(\Phi \cap[0,1]^{d}\right)=0,
$$

where the $d x$ always refers to integration with respect to the Lebesgue measure on the underlying space (in this case $\mathbb{R}^{d}$ ). The intensity measure $\Lambda$ of a stationary point process $\Phi$ fulfills

$$
\Lambda(A)=\gamma V_{d}(A)
$$

for all Borel sets $A$, where the intensity $\gamma$ of $\Phi$ is defined as the mean number of points falling in a set of volume 1 , i.e.

$$
\gamma:=\mathbb{E} \Phi\left([0,1]^{d}\right)
$$

We always assume $\gamma<\infty$.

The contributions by C. Beisbart et al. and by V. Robins in Chap. 3, and by G. Döge and D. Stoyan in Chap. 4 of this volume contain applications of point processes in the natural sciences.

\subsection{Particle Processes}

Point processes can be considered on quite general metric spaces. In particular, we can define a point process $X$ on $\mathcal{C}^{d}$, the class of compact subsets of $\mathbb{R}^{d}$, to be a locally finite random subset of $\mathcal{C}^{d}$ (equivalently, a locally finite, random counting measure on $\mathcal{C}^{d}$ ). Such a point process $X$ is called a particle process. In the present context, $X$ is said to be locally finite if

$$
X\left(\left\{C \in \mathcal{C}^{d}: C \cap D \neq \emptyset\right\}\right)<\infty, \quad D \in \mathcal{C}^{d}
$$

Condition (11) is stronger than $X(A)<\infty$, for each compact subset $A \subset \mathcal{C}^{d}$ (here compactness refers to the Hausdorff metric on $\mathcal{C}^{d}$ ).

More general processes are possible where the 'particles' are closed sets, for example affine subspaces (flats) of a certain dimension $k \in\{0, \ldots, d-1\}$ (the case $k=0$ brings 
us back to the ordinary point processes on $\mathbb{R}^{d}$ ). Although contact distributions can also be considered for such $k$-flat processes, we will concentrate on compact particles, in the following.

Since the translation group operates on $\mathcal{C}^{d}$ in the obvious way, we can define invariance properties of particle processes $X$. In particular, we call $X$ stationary, if the distribution of $X$ is translation invariant.

\subsection{Particle Processes as Marked Point Processes}

In general, a marked point process $\Psi$ on $\mathbb{R}^{d}$ (with a suitable metric space $\mathbf{K}$ as mark space) is a point process on $\mathbb{R}^{d} \times \mathbf{K}$ such that $\Psi(C \times \mathbf{K})<\infty$ almost surely for any compact set $C \subset \mathbb{R}^{d}$. The points of $\Psi$ can be enumerated in the form $\left(\xi_{1}, \kappa_{1}\right),\left(\xi_{2}, \kappa_{2}\right), \ldots$, where the $\xi_{n}$ are random elements of $\mathbb{R}^{d}$ and the $\kappa_{n}$ are random elements of $\mathbf{K}$. Here we will only consider marked point processes for which $\xi_{n} \neq \xi_{m}$ for $n \neq m$, that is, for which the unmarked point process $\Phi:=\Psi(\cdot \times \mathbf{K})$ is simple. The intensity measure of a marked point process is a measure on $\mathbb{R}^{d} \times \mathbf{K}$. Its value on a Borel set $A \subset \mathbb{R}^{d} \times \mathbf{K}$ is again the expected number $\mathbb{E} \Psi(A)$ of points of $\Psi$ falling into $A$. In case $A=B \times L$, this is the mean number of marked points $(x, K) \in \Psi$ with $x \in A$ and mark $K \in L$. A marked point process is said to be stationary if the distribution of $\left\{\left(\xi_{n}+x, \kappa_{n}\right): n=1,2, \ldots\right\}$ is independent of $x \in \mathbb{R}^{d}$. In that case we define the intensity $\gamma$ of $\Psi$ as the intensity of the unmarked process $\Phi$. The intensity measure of a stationary marked point process $\Psi$ with finite intensity $\gamma$ is of the form $\mathbb{E} \Psi(\cdot)=\gamma V_{d} \otimes \mathbb{Q}$, where $\mathbb{Q}$ is a probability measure on $\mathbf{K}$. If the marks are independent and identically distributed as well as independent of the point process $\Phi$, we say that $\Psi$ is an independent marking of $\Phi$ or (somewhat sloppy) that $\Psi$ has independent marks. In this case we have the decomposition $\mathbb{E} \Psi(\cdot)=\Lambda \otimes \mathbb{Q}$, where $\Lambda$ is the intensity measure of $\Phi$ and $\mathbb{Q}$ is the distribution of the marks. Notice that, in general, stationarity does not imply independent marks; an important example where this implication holds is the Poisson process discussed in later sections. In the cases where $\Psi$ is stationary or obtained by independent marking, we call $\mathbb{Q}$ the mark distribution of $\Psi$.

Physical applications of marked point processes can be found in the contribution by C. Beisbart, M. Kerscher and K. Mecke in Chap. 4 of this volume, as well as in the article by F. Schmid and N.H. Phuong in Chap. 2.

There is an important connection between particle processes and marked point processes with mark space $\mathcal{C}^{d}$ (instead of marks we speak of grains here). Each particle process $X$ can be transformed into a marked point process $\Psi$ on $\mathbb{R}^{d} \times \mathcal{C}^{d}$, if compact sets $C$ are represented as pairs $(x, D)$ (where we think of $x$ as the 'location' of $C$ and where $D:=C-x$ is considered to be the 'shape' of $C$ ). Such a representation is always possible, if we have chosen a center map $c: \mathcal{C}^{d} \rightarrow \mathbb{R}^{d}$, that is a measurable mapping which is translation covariant in the sense that $c(C+x)=c(C)+x$ is satisfied, for $x \in \mathbb{R}^{d}$ and $C \in \mathcal{C}^{d} \backslash\{\emptyset\}$, and $c(\emptyset)=0$. For a given center map $c$, the mapping $C \mapsto(c(C), C-c(C))$ transforms $X$ into a marked point process $\Psi$. Here we assume that different particles have different centers. (The finiteness condition for marked point processes is another extra assumption; if $c(C) \in C$ for all $C \in X$, then it is induced by (1).) The marked point process $\Psi$ is stationary if and only if $X$ is stationary. Further, in the stationary case, the grain distribution $\mathbb{Q}$ is concentrated on $\mathcal{C}_{0}^{d}:=\left\{C \in \mathcal{C}^{d}: c(C)=0\right\}$. 
Also then the intensity of $\Psi$ can be interpreted as the mean number of particles of $X$ per unit volume.

The representation of a particle process as a marked point process is frequently suggested by the given physical situation, and then a center map is given in a natural way. For example, we may choose $c(C)$ as the midpoint of the circumsphere of $C$ in which case the particles $C+x,(x, C) \in \Psi$, can be interpreted as grains grown around the germs $x$. A further center map of physical nature is the center of mass. In statistical applications often other center maps are natural, for example the lower/upper left tangent point. In the stationary case, different representations of a particle process $X$ as marked point processes $\Psi_{1}, \Psi_{2}$ (according to different center maps $c_{1}, c_{2}$ ) lead to the same intensities, but to different (though closely related) grain distributions $\mathbb{Q}_{1}, \mathbb{Q}_{2}$. In the following it is convenient to represent a particle process $X$ as

$$
X=\{C+x:(x, C) \in \Psi\}=\left\{Z_{n}+\xi_{n}: n \in \mathbb{N}\right\}
$$

where $\Psi=\left\{\left(\xi_{n}, Z_{n}\right): n \in \mathbb{N}\right\}$ is a marked point process which is locally finite, i.e. satisfies the condition

$$
\int_{\mathbb{R}^{d} \times \mathcal{C}^{d}} 1\{(C+x) \cap D \neq \emptyset\} \Psi(d(x, C))<\infty, \quad D \in \mathcal{C}^{d},
$$

equivalent to (1).

\subsection{Random Sets and Grain Models}

Point processes in $\mathbb{R}^{d}$ can be interpreted as locally finite random sets. Now we consider more general random (closed) sets $Z$ in $\mathbb{R}^{d}$. Formally, a random closed set $Z$ can be defined as a random element of the space $\mathcal{F}^{d}$ of all closed subsets of $\mathbb{R}^{d}$ (again, a mathematical description requires a suitable $\sigma$-field on $\mathcal{F}^{d}$ such that $Z$ becomes a measurable mapping $Z: \Omega \rightarrow \mathcal{F}^{d}$ ). For the general theory, see [24, 39, 42]. We say that $Z$ is stationary, if $Z$ and $Z+x$ have the same distribution, for all $x \in \mathbb{R}^{d}$. A general example of a random set $Z$ is the union set of a particle process $X$ or, equivalently,

$$
Z=\bigcup_{n \in \mathbb{N}}\left(Z_{n}+\xi_{n}\right)
$$

where $\Psi=\left\{\left(\xi_{n}, Z_{n}\right): n \in \mathbb{N}\right\}$ is the marked point process representing $X$ as in (2). Condition (3) (resp. (1)) guarantees that $Z$ is closed. A random set $Z$ represented as in (4) is also referred to as a grain model. The reader should be warned that grain models have been defined in different ways in the literature (see e.g. [29, 39, 42]).

Since a non-empty stationary random closed set $Z$ is almost surely either empty or its convex hull coincides with the whole space $\mathbb{R}^{d}$, a stationary grain model is almost surely either empty or the union of infinitely many grains.

Grain models are still of quite general nature. In fact, every random closed set $Z$ in $\mathbb{R}^{d}$ allows a representation (4). Moreover, if $Z$ is stationary, one can assume that the marked point process $\Psi$ is also stationary. In order to get a smaller (and more interesting) class, we may assume that $\Psi$ has independent marks, that means, the grains $Z_{1}, Z_{2}, \ldots$ 
are independent and identically distributed as well as independent of the germ process $\Phi$. In this case, or if $\Psi$ is stationary, we call the mark distribution $\mathbb{Q}$ the distribution of the typical grain and fix a random closed set $Z_{0}$ (the typical grain) with this distribution. The most prominent example of a grain model with independent grains is the Boolean model, where the underlying germ process $\Phi$ is a Poisson process. In the next section, we study this class of random sets (and point processes) in more detail under the assumption of stationarity; more general point processes and random sets are discussed subsequently.

\subsection{Grain Models with Convex Grains}

Of special interest are random sets $Z$ with realizations in the extended convex ring

$$
\mathcal{S}^{d}:=\left\{A \in \mathcal{F}^{d}: A \cap K \in \mathcal{R}^{d} \text { for all } K \in \mathcal{K}^{d}\right\} .
$$

Here, $\mathcal{K}^{d}$ denotes the class of all compact convex subsets of $\mathbb{R}^{d}$ (convex bodies), and the convex ring $\mathcal{R}^{d}$ is the class of finite unions of convex bodies (polyconvex bodies). Random $\mathcal{S}^{d}$-sets provide a sufficiently general framework to cover most situations which arise in practical applications (see [32. 42]). If a grain model $Z$ is given for which all the grains are convex, then $Z$ is a random $\mathcal{S}^{d}$-set. This model is of particular importance, since every random $\mathcal{S}^{d}$-set $Z$ can be represented as a grain model (4) with convex grains $Z_{i}$ (and with stationary germ process $\Phi$, if $Z$ is stationary) (see [39] and [52]). Whereas the representation of $Z$ as a grain model with compact grains is rather simple, it is more difficult to construct a decomposition into random convex grains which have the same invariance properties as $Z$.

\subsection{Integrability Conditions}

For a grain model $Z$, the assumption (3) on the underlying marked point process $\Psi$ (or the corresponding particle process (2) is implied by

$$
\int_{\mathbb{R}^{d} \times \mathcal{C}^{d}} 1\{(C+x) \cap D \neq \emptyset\} \tilde{\Lambda}(d(x, C))<\infty, \quad D \in \mathcal{C}^{d},
$$

where $\tilde{\Lambda}$ is the intensity measure of $\Psi$. By Campbell's theorem, the above integral is just the expectation of (3). In the case of stationarity, condition (5), for all $D \in \mathcal{C}^{d}$, amounts to the same as requiring that the typical grain $Z_{0}$ fulfills

$$
\mathbb{E} V_{d}\left(Z_{0}+D\right)<\infty, \quad D \in \mathcal{C}^{d}
$$

(see also [8] or [39]).

\section{Contact Distribution Functions}

\subsection{The Capacity Functional}

By a simple measure theoretic argument, one sees that the distribution of a random set $Z$ is completely determined by the capacity functional $T_{Z}$ of $Z$. It is defined on $\mathcal{C}^{d}$ by 


$$
T_{Z}(C):=\mathbb{P}(Z \cap C \neq \emptyset)=1-\mathbb{P}(Z \cap C=\emptyset), \quad C \in \mathcal{C}^{d} .
$$

In the case of a point process $\Phi$ on $\mathbb{R}^{d}$ we have $T_{\Phi}(C)=1-\mathbb{P}(\Phi(C)=0)$ for $C \in \mathcal{C}^{d}$. The distribution of a random closed set $Z$ need not be determined by the restriction of $T_{Z}$ to $\mathcal{K}^{d}$ (this fact applies already to point processes). However, the values $T_{Z}(K), K \in \mathcal{K}^{d}$, still contain interesting information and are easier to treat from both an analytical and a statistical point of view. They are closely connected to the contact distribution functions of $Z$ which we introduce next.

\subsection{Contact Distributions}

Let $B \in \mathcal{K}^{d}$ with $0 \in B$ be fixed. For a stationary random set $Z$ the formal definition of the contact distribution function with structuring element $B$ is

$$
H_{B}(r):=\mathbb{P}(r B \cap Z \neq \emptyset \mid 0 \notin Z), \quad r \geq 0,
$$

or equivalently,

$$
H_{B}(r)=\mathbb{P}\left(d_{B}(0, Z) \leq r \mid 0 \notin Z\right), \quad r \geq 0,
$$

(we always assume $\mathbb{P}(0 \notin Z)>0$, i.e. we exclude the uninteresting case $Z=\mathbb{R}^{d} \mathbb{P}$-a.s., where the contact distribution is not well defined). $H_{B}$ is a distribution function in the extended sense, it may have an atom at infinity.

A closely related concept is the empty space function

$$
F_{B}(r):=\mathbb{P}\left(d_{B}(0, Z) \leq r\right)=T_{Z}(r B), \quad r \geq 0,
$$

of $Z$. It has an atom at 0 of size

$$
p:=\mathbb{P}(0 \in Z)
$$

Due to stationarity and Fubini's theorem we have

$$
p=\int_{[0,1]^{d}} \mathbb{P}(x \in Z) d x=\mathbb{E} V_{d}\left([0,1]^{d} \cap Z\right),
$$

which explains why $p$ is called the volume fraction of $Z$ (the mean volume of $Z$ per unit volume of $\mathbb{R}^{d}$ ). Since $0 \in B$ we have

$$
(1-p)\left(1-H_{B}(r)\right)=\mathbb{P}(r B \cap Z=\emptyset)=1-F_{B}(r) .
$$

If $p=0$ (this occurs for lower dimensional sets $Z$ ), $H_{B}$ and $F_{B}$ coincide.

For a given realization of $Z, H_{B}$ can be estimated from volume measurements. Namely, we have

$$
\begin{aligned}
H_{B}(r) & =\mathbb{P}\left(d_{B}(0, Z) \leq r \mid 0 \notin Z\right)=\mathbb{P}(r B \cap Z \neq \emptyset \mid 0 \notin Z) \\
& =1-\frac{\mathbb{P}\left(0 \notin Z+r B^{*}\right)}{\mathbb{P}(0 \notin Z)}=\frac{\mathbb{P}\left(0 \in Z+r B^{*}\right)-p}{1-p} \\
& =\frac{\mathbb{E}\left[V_{d}\left(\left(Z+r B^{*}\right) \cap[0,1]^{d}\right)-p\right]}{1-p}, \quad r \geq 0 .
\end{aligned}
$$


A straightforward generalization is

$$
H_{B}(r)=\frac{\mathbb{E}\left[V_{d}\left(\left(Z+r B^{*}\right) \cap W\right)-V_{d}(Z \cap W)\right]}{V_{d}(W)-\mathbb{E} V_{d}(Z \cap W)},
$$

for an arbitrary Borel set $W \subset \mathbb{R}^{d}$ of positive volume.

If $Z=\Phi$ is a point process, we have $p=0$, hence the condition $0 \notin \Phi$ (required in the definition of a contact distribution function) is fullfilled almost surely. Also, in this case, $H_{B} \equiv 0$ if $B$ is lower dimensional. If $B$ has a non-empty interior, then

$$
(1-p)\left(1-H_{B}(\infty)\right)=\mathbb{P}(Z=\emptyset)
$$

where $H_{B}(\infty):=\lim _{t \rightarrow \infty} H_{B}(t)$. Hence $H_{B}(\infty)<1$ in case $\mathbb{P}(Z=\emptyset)>0$. For point processes there exist notions related to the contact distribution function such as the nearest-neighbour distribution function or the $J$-function introduced in [47] and extended in [48]; see also [42]. For the latter, an extension to certain grain models is suggested in [20]. Other extensions concern the $n$-neighbour distance distribution, see [16] and [44] for further details.

The values of the contact distribution functions $H_{B}$ essentially depend on the choice of the gauge body $B$. Therefore, we may choose different gauge bodies to gain additional information about a given random structure. Two contact distribution functions, which have received particular attention in the literature, are the spherical contact distribution function $H_{s}:=H_{B^{d}}$ and the linear contact distribution function $H_{[0, u]}$ in direction $u \in S^{d-1}$, where $S^{d-1}:=\partial B^{d}$ is the Euclidean unit sphere (in the literature often $H_{l}$ is used for the linear contact distribution; however, this does not reflect the dependence on the direction $u$ which is important if $Z$ is not isotropic). The connection of the linear contact distribution to chord length distributions is explained in [42]; see also the references given in Sect. 12 For an application of this connection to the study of contact and chord length distributions of stationary Voronoi tessellations see [10] and [31]. The following sections contain a more detailed discussion of these special contact distributions.

\subsection{Estimators of Contact Distribution Functions}

Formula (9) suggests

$$
\frac{V_{d}\left(\left(Z+r B^{*}\right) \cap W\right)-V_{d}(Z \cap W)}{V_{d}(W)-V_{d}(Z \cap W)}
$$

as a simple estimator of $H_{B}(r)$ (plus sampling). It requires, however, knowledge from outside the window $W$. A traditional approach to cope with this problem is to consider only those points in $W$ having a certain minimal distance from the boundary of $W$ (minus sampling). Further information on this and more sophisticated methods can be found in [1, 42, 45]. In [3] an instructive comparison is provided between Hanisch type (HorvitzThompson style) and Kaplan-Meier type estimators for the empty space function of a stationary random closed set. 
The estimators of $H_{B}$ discussed in the literature are based on a single observation of $Z$ and can be computed (or rather approximated) with the help of digital image analysers. They serve as convenient and useful summary statistics in the exploratory analysis of spatial patterns (see e.g. [2, 3, 6, 7, 9]). For instance one might plot these estimators against theoretically known contact distribution functions, such as those of the Boolean model with convex grains (see Sect. 5). If $Z$ is ergodic, then $H_{B}$ can in principle be determined from a single realization of $Z$ by extending the observation window (see [9]).

\subsection{Regularity Properties}

The contact distribution functions of stationary random closed sets with respect to general gauge bodies enjoy an important regularity property. In [7] it was shown that the contact distribution function $H_{B}$ of a stationary random closed set $Z$ is absolutely continuous if 0 is an interior point of the gauge body $B$. Hence it makes sense to define and estimate the density (and the hazard rate). More specifically, in the spherical case the density $H_{s}^{\prime}$ of $H_{s}$ satisfies

$$
(1-p) H_{s}^{\prime}(r)=\mathbb{E}\left[\mathcal{H}^{d-1}\left(\partial\left(Z+r B^{d}\right) \cap[0,1]^{d}\right)\right], \quad r>0,
$$

where $\mathcal{H}^{d-1}(C)$ is the $(d-1)$-dimensional Hausdorff measure of $C$. (If $C$ is a smooth $(d-1)$-dimensional surface, then $\mathcal{H}^{d-1}(C)$ is just the surface area of $C$.) Independently, and by a completely different approach, in [21] not only the absolute continuity of $H_{s}$ for a random $\mathcal{S}^{d}$-set was proved, but also a formula expressing the density in terms of certain geometric mean values. We will resume this issue later in this paper.

\subsection{Generalizations of Contact Distributions}

We have already mentioned that the contact distribution functions do not determine the distribution of $Z$. Hence, it is natural to extend their definition by taking into account more information than just the distance $d_{B}(0, Z)$. Using the contact direction vector $u_{B}(0, Z)$, we can define a generalized contact distribution function of $Z$,

$$
H_{B}(r, C):=\mathbb{P}\left(d_{B}(0, Z) \leq r, u_{B}(0, Z) \in C \mid 0 \notin Z\right),
$$

where $r \geq 0$ and $C \subset \partial B$ is a Borel set. This definition is subject to the assumption that the vector $u_{B}(0, Z)$ is $\mathbb{P}$-a.s. well-defined given that $0 \notin Z$. If $Z$ is a random closed set and $B$ is strictly convex, containing 0 in its interior, then this is indeed the case. This follows from $V_{d}\left(\operatorname{exo}_{B}(Z)\right)=0$ (compare the remark at the end of Sect. 2) and the stationarity of $Z$. The function $H_{B}(\cdot, \cdot)$ determines the conditional distribution of the pair $\left(d_{B}(0, Z), u_{B}(0, Z)\right)$ and hence that of the contact vector $d_{B}(0, Z) u_{B}(0, Z)$. For each fixed $r$ the function $H_{B}(r, \cdot)$ is a measure on $\partial B$. Note that $H_{B}(\cdot, \partial B)$ is the contact distribution function defined by $(7)$.

In Sect. 11 further extensions of (generalized) contact distributions are discussed; there, in particular, local (second order) information about $Z$ at the point of contact is taken into account. 


\section{Poisson Processes and Boolean Models}

In this section we provide explicit formulas for contact distributions of stationary Poisson processes and Boolean models with respect to various structuring elements $B$. Applications of these models in physics may be found in [25, 26] or in the contributions by $\mathrm{C}$. Arns, M. Knackstedt and K. Mecke (Chap. 1) and by V. Robins (Chap. 3) in this volume.

\subsection{The Stationary Poisson Process}

The most important instance of a stationary point process on $\mathbb{R}^{d}$ is a stationary Poisson process. It is the mathematical model for a collection of points that are distributed "completely random" in space. For a Poisson process $\Phi$ on $\mathbb{R}^{d}, \Phi\left(A_{1}\right), \ldots, \Phi\left(A_{k}\right)$ are stochastically independent random variables, whenever the measurable sets $A_{1}, \ldots, A_{k} \subset \mathbb{R}^{d}$ are pairwise disjoint. Moreover,

$$
\mathbb{P}(\Phi(A)=m)=\frac{\left(\gamma V_{d}(A)\right)^{m}}{m !} \exp \left[-\gamma V_{d}(A)\right], \quad m=0,1, \ldots,
$$

for all Borel sets $A \subset \mathbb{R}^{d}$, i.e. $\Phi(A)$ is Poisson distributed with parameter $\gamma V_{d}(A)$. It follows that $\gamma$ is the intensity of $\Phi$ and that the distribution of a stationary Poisson process $\Phi$ is completely determined by its intensity.

From the uniqueness result mentioned in the previous section we obtain that a point process $\Phi$ on $\mathbb{R}^{d}$ is a stationary Poisson process of intensity $\gamma$ if and only if

$$
\mathbb{P}(\Phi(A)=0)=\exp \left[-\gamma V_{d}(A)\right]
$$

for all Borel sets $A \subset \mathbb{R}^{d}$. In this case we have

$$
H_{B}(r)=1-\mathbb{P}(\Phi(r B)=0)=1-\exp \left[-\gamma V_{d}(B) r^{d}\right], \quad r \geq 0 .
$$

\subsection{The Stationary Boolean Model}

A stationary Boolean model $Z$ is a grain model

$$
Z=\bigcup_{n \in \mathbb{N}}\left(Z_{n}+\xi_{n}\right)
$$

with independent, identically distributed grains $Z_{n}$, where the underlying point process $\Phi=\left\{\xi_{n}: n=1,2, \ldots\right\}$ is a stationary Poisson process that is independent of $\left\{Z_{n}: n=\right.$ $1,2 \ldots\}$. In this case the finiteness condition (3) is even equivalent to 6 . An alternative definition of the Boolean model in terms of a stationary Poisson particle process $X$ will be given later in Sect. 10.3

The distribution of a stationary Boolean model $Z$ is determined by the pair $(\gamma, \mathbb{Q})$, where $\gamma$ is the intensity of $\Phi$ and $\mathbb{Q}$ is the common distribution of the grains $Z_{i}$. We again consider a random set $Z_{0}$ with distribution $\mathbb{Q}$ and call this the typical grain of $Z$. The capacity functional $T_{Z}$ of $Z$ has the form 


$$
\begin{aligned}
T_{Z}(C) & =1-\mathbb{P}(Z \cap C=\emptyset) \\
& =1-\exp \left\{-\gamma \int_{\mathcal{C}^{d}} V_{d}\left(K+C^{*}\right) \mathbb{Q}(d K)\right\} \\
& =1-\exp \left\{-\gamma \mathbb{E} V_{d}\left(Z_{0}+C^{*}\right)\right\}
\end{aligned}
$$

and hence we have

$$
p=\mathbb{P}(0 \in Z)=1-\exp \left\{-\gamma \mathbb{E} V_{d}\left(Z_{0}\right)\right\}
$$

Reversely the distribution of $Z$ determines the intensity $\gamma$ and the distribution $\mathbb{Q}$ (up to the centering of the particles, see [39]). The stationary Boolean model will be generalized later in this paper. If not stated otherwise a Boolean model will always be assumed to be stationary.

\subsection{The Spherical Contact Distribution of the Boolean Model}

By (13) the spherical contact distribution $H_{s}$ of the stationary Boolean model $Z$ is given by

$$
H_{s}(r)=1-\exp \left\{-\gamma \int_{\mathcal{C}^{d}}\left[V_{d}\left(K+r B^{d}\right)-V_{d}(K)\right] \mathbb{Q}(d K)\right\} .
$$

At this point, we can proceed further if we assume convexity of the grains, since this allows us to use the Steiner formula for convex bodies,

$$
V_{d}\left(K+r B^{d}\right)=\sum_{j=0}^{d} r^{j} b_{j} V_{d-j}(K) .
$$

Here, $b_{j}$ is the ( $j$-dimensional) volume of the Euclidean unit ball in $\mathbb{R}^{j}$ and the coefficients $V_{0}(K), \ldots, V_{d}(K)$ are the intrinsic volumes or Minkowski functionals of the convex body $K$. Note that these functionals occur in the literature with different normalization, different enumeration and also different names. We have chosen here the notation which is standard in convex geometry (see Schneider's book 37]). In particular, $V_{d}(K)$ is the volume of $K, V_{d-1}(K)$ is half the surface area, $V_{d-2}(K)$ is proportional to the integral mean curvature, ..., $V_{1}(K)$ is proportional to the mean width of $K$, and $V_{0}(K)$ is the Euler characteristic (which, for convex $K$, is 1 if $K$ is non-empty and 0 if $K$ is the empty set). Combining (14) with (15), we arrive at

$$
H_{s}(r)=1-\exp \left\{-\gamma \sum_{j=1}^{d} r^{j} b_{j} \mathbb{E} V_{d-j}\left(Z_{0}\right)\right\} .
$$

The usefulness of (16) for the statistical analysis of $Z$ is obvious. Fitting a polynomial of order $d$ (and without constant term) to the (empirical) function corresponding to $-\ln \left(1-H_{s}(r)\right)$, yields estimators for $\gamma \mathbb{E} V_{0}\left(Z_{0}\right), \ldots, \gamma \mathbb{E} V_{d-1}\left(Z_{0}\right)$ and can also be used to check the Boolean hypothesis (see [40] or [42]). Note that here $\gamma \mathbb{E} V_{0}\left(Z_{0}\right)=\gamma$, hence we obtain in particular an estimator of the intensity $\gamma$ of $\Phi$. Note also that the remaining mean value $\gamma \mathbb{E} V_{d}\left(Z_{0}\right)$ can be estimated directly from the volume fraction $p$ of $Z$. 


\subsection{General Structuring Elements}

If we aim to estimate other functionals of the stationary Boolean model $Z$, in particular, functionals which reflect the anisotropy of $Z$, then the spherical contact distribution $H_{s}$ is no longer sufficient and we are forced to use more general structuring elements $B$ (e.g. linear contact distributions $H_{B}$ where $B=[0, u]$ is a segment). The above formula for the capacity functional of a Boolean model (with convex grains) immediately implies

$$
H_{B}(r)=1-\exp \left\{-\gamma \int_{\mathcal{K}^{d}}\left[V_{d}\left(K+r B^{*}\right)-V_{d}(K)\right] \mathbb{Q}(d K)\right\} .
$$

For a further investigation of the right side, we again assume convexity, not only of the grains $K$, but also of $B$. The reason for this is that if $B$ is a convex body, then a generalization of the Steiner formula can be used. More explicitly, given convex bodies $K, B$, the generalized Steiner formula reads

$$
V_{d}(K+B)=\sum_{k=0}^{d}\left(\begin{array}{l}
d \\
k
\end{array}\right) V(K[k], B[d-k])
$$

with mixed volumes

$$
V(K[k], B[d-k]):=V(\underbrace{K, \ldots, K}_{k}, \underbrace{B, \ldots, B}_{d-k})
$$

(sometimes we write $V(K, B[d-1])$ instead of $V(K[1], B[d-1]))$. Hence, in this case, we obtain

$$
H_{B}(r)=1-\exp \left\{-\gamma \sum_{k=1}^{d}\left(\begin{array}{l}
d \\
k
\end{array}\right) r^{k} \int_{\mathcal{K}^{d}} V\left(K[d-k], B^{*}[k]\right) \mathbb{Q}(d K)\right\} .
$$

Relation (16) is a special case of (18) as can be seen from

$$
V_{j}(K)=\frac{\left(\begin{array}{l}
d \\
j
\end{array}\right)}{b_{d-j}} V\left(K[j], B^{d}[d-j]\right), \quad j=0, \ldots, d .
$$

Therefore measurements of the contact distribution function $H_{B}(r), r \geq 0$, for convex bodies $B \in \mathcal{K}^{d}$ as structuring elements lead to estimators for

$$
\gamma \int_{\mathcal{K}^{d}} V\left(K[d-k], B^{*}[k]\right) \mathbb{Q}(d K), \quad k=1, \ldots, d .
$$

These formulas can be specified further by special choices of $B$ and under additional assumptions on the grains. For instance, one can choose $B$ to be a cube and the typical grain to be $\mathbb{P}$-a.s. a rectangular parallelepiped; compare [45]. 


\subsection{The Linear Contact Distribution of the Boolean Model}

The linear contact distribution functions are defined with a gauge body $B$ that is a segment $[0, u], u \in S^{d-1}$. For $B=[0, u]$, the summands in $[18]$ corresponding to $k \geq 2$ all vanish, and hence

$$
\begin{aligned}
H_{[0, u]}(r) & =1-\exp \left\{-\gamma r d \int_{\mathcal{K}^{d}} V([0, u], K[d-1]) \mathbb{Q}(d K)\right\} \\
& =1-\exp \left\{-\gamma r \int_{\mathcal{K}^{d}} V_{d-1}\left(K \mid u^{\perp}\right) \mathbb{Q}(d K)\right\} \\
& =1-\exp \left\{-\gamma \frac{r}{2} \int_{\mathcal{K}^{d}} \int_{S^{d-1}}|\langle u, v\rangle| S_{d-1}(K ; d v) \mathbb{Q}(d K)\right\}
\end{aligned}
$$

due to classical formulas for the mixed volume $V([0, u], K[d-1])$ (compare $\S 5.3$ in [37]). Here, $V_{d-1}\left(K \mid u^{\perp}\right)$ is the $(d-1)$-volume of the orthogonal projection of $K$ onto the hyperplane $u^{\perp},\langle u, v\rangle$ is the scalar product of the (unit) vectors $u, v$, and $S_{d-1}(K ; \cdot)$ denotes the surface area measure of $K$ (of order $d-1$ ), which is a finite measure on $S^{d-1}$ (see Sect.7). If we define the mean surface area measure of the typical grain by

$$
\bar{S}_{d-1}:=\int_{\mathcal{K}^{d}} S_{d-1}(K ; \cdot) \mathbb{Q}(d K)
$$

as a measure over $S^{d-1}$, we finally obtain

$$
H_{[0, u]}(r)=1-\exp \left\{-\gamma \frac{r}{2} \int_{S^{d-1}}|\langle u, v\rangle| \bar{S}_{d-1}(d v)\right\} .
$$

If $Z$ (and hence $Z_{0}$ ) is isotropic, $\mathbb{Q}$ is rotation invariant, and therefore $H_{l}:=H_{[0, u]}$ is independent of $u$. We obtain

$$
H_{l}(r)=1-\exp \left\{-\gamma r \frac{2 b_{d-1}}{d b_{d}} \mathbb{E} V_{d-1}\left(Z_{0}\right)\right\} .
$$

\subsection{The Mean Normal Distribution}

The measure $\mu:=\gamma \bar{S}_{d-1}$ is also called the oriented mean normal measure of the underlying Poisson particle process $X, X:=\left\{Z_{1}+\xi_{1}, Z_{2}+\xi_{2}, \ldots\right\}$ (with $\Phi=$ $\left\{\xi_{1}, \xi_{2}, \ldots\right\}$ and with convex grains $Z_{i}$ ). The oriented mean normal measure of $X$ is the surface area measure of a convex body (unique up to translations, if $\mu$ is not degenerated), which is called the Blaschke body $B(X)$ of $X$. The normalized measure

$$
\mathbb{M}:=\frac{\mu}{\mu\left(S^{d-1}\right)}=\frac{\mathbb{E} S_{d-1}\left(Z_{0} ; \cdot\right)}{2 \mathbb{E} V_{d-1}\left(Z_{0}\right)}
$$

is called oriented mean normal distribution, it describes the distribution of the outer normal vector in a typical boundary point of the grains (more precisely, a typical point of $\left.\bigcup_{n=1}^{\infty}\left(\partial Z_{n}+\xi_{n}\right)\right)$. We emphasize that $\mathbb{M}$ contains oriented directional information 
about the grains, it distinguishes between outer and inner normals in the boundary points. The corresponding unoriented mean normal distribution is given by

$$
\widetilde{\mathbb{M}}(A):=\frac{1}{2}\left(\mathbb{M}(A)+\mathbb{M}\left(A^{*}\right)\right), \quad A \subset S^{d-1} .
$$

If the grains are almost surely $(d-1)$-dimensional, then the oriented and the unoriented mean normal distributions coincide. They both vanish if the grains have dimension $\leq d-2$. However, for Boolean models $Z$ with full dimensional grains (or surfaces as grains), the measures $\mathbb{M}$ and $\widetilde{\mathbb{M}}$ contain relevant directional information about the grains. It is therefore important to know, how far the measures $\widetilde{\mathbb{M}}$ (resp. $\mathbb{M}$ ) are determined by linear contact distribution functions $H_{[0, u]}, u \in S^{d-1}$. Since

$$
H_{[0, u]}(r)=1-\exp \left\{-\gamma r \mathbb{E} V_{d-1}\left(Z_{0}\right) \int_{S^{d-1}}|\langle u, v\rangle| \widetilde{\mathbb{M}}(d v)\right\},
$$

we find that the collection of all linear contact distribution functions $\left\{H_{[0, u]}, u \in S^{d-1}\right\}$ determines the unoriented mean normal distribution $\widetilde{\mathbb{M}}$ uniquely (but not the oriented mean normal distribution $\mathbb{M}$ ). The reason for this result is that the spherical integral transform (the so-called Cosine transform)

$$
\rho \mapsto T \rho, \quad T \rho(u):=\int_{S^{d-1}}|\langle u, v\rangle| \rho(d v),
$$

which maps finite measures $\rho$ on $S^{d-1}$ to continuous functions, is injective on even measures (but is not injective in general, since $T \rho=T \rho^{*}$, where $\rho^{*}$ is the reflection of $\rho$ ). If we restrict $T$ to the even $C^{\infty}$ functions, then the inverse transform $T^{-1}$ can be obtained using expansions into spherical harmonics. However, the inverse transform is not stable, which means that it is quite sensitive to small perturbations of the given data. For example, if the linear contact distributions $H_{[0, u]}(r)$ of $Z$ are determined for a fixed $r$ and finitely many directions $u=u_{1}, \ldots, u_{k}$, and if the resulting values $H_{\left[0, u_{1}\right]}(r), \ldots, H_{\left[0, u_{k}\right]}(r)$ are interpolated to obtain a smooth function $f$ on $S^{d-1}$, the resulting estimator

$$
-\frac{1}{\gamma r \mathbb{E} V_{d-1}\left(Z_{0}\right)} T^{-1} \ln (1-f(\cdot))
$$

for $\widetilde{\mathbb{M}}$ will typically be a signed measure, which may be far from the true one. This problem can be overcome by means of more sophisticated estimators (based on linear programming methods or the EM algorithm), it is discussed in a quite similar situation (the estimation of fibre processes from hyperplane sections) in [17].

\section{Poisson Cluster Processes}

Poisson processes can be used to build more general point process models. An important example are Poisson cluster processes whose spherical contact distribution functions are studied in the present section. This subject is continued in Sect. 8 with the investigation of Poisson cluster models. 


\subsection{Definition of a Poisson Cluster Process}

We start with the construction of Poisson cluster processes. There is a stationary Poisson process $\Phi_{p}$ (of parents) with finite intensity $\gamma_{p}$ and a family $\left\{N_{x}: x \in \Phi_{p}\right\}$ of finite point processes on $\mathbb{R}^{d}$ such that $\left\{\left(x, N_{x}\right): x \in \Phi_{p}\right\}$ is an independent marking of $\Phi_{p}$. The conditional distribution of $N_{x}$ given $\Phi_{p}$ is the same for all $x \in \Phi_{p}$ and we let $N_{0}$ denote a typical cluster, i.e. a point process with this distribution. We also assume that the mean number $\gamma_{c}$ of cluster points,

$$
\gamma_{c}:=\mathbb{E} N_{0}\left(\mathbb{R}^{d}\right)
$$

is finite. Then

$$
\Phi:=\bigcup_{x \in \Phi_{p}}\left(N_{x}+x\right)
$$

is a stationary point process with intensity

$$
\gamma=\gamma_{p} \gamma_{c}
$$

It is called Poisson cluster process based on $\Phi_{p}$ and the typical cluster $N_{0}$ (we remark that the points of different clusters are almost surely different, i.e. we do not have multiple points).

\subsection{Contact Distributions of a Poisson Cluster Process}

A Poisson cluster process $\Phi$ can be interpreted as a Boolean model with typical (compact but in general not convex) grain $N_{0}$. Using (13), we obtain that

$$
T_{\Phi}(C)=1-\exp \left\{-\gamma_{p} \mathbb{E} V_{d}\left(N_{0}+C^{*}\right)\right\}
$$

for $C \in \mathcal{C}^{d}$. This shows that the distribution of $\Phi$ is determined by $\gamma_{p}$ and by the distribution of $N_{0}$. In particular, the contact distribution of $\Phi$ with respect to a fixed structuring element $B$ is given by

$$
H_{B}(r)=1-\exp \left\{-\gamma_{p} \mathbb{E} V_{d}\left(N_{0}+r B^{*}\right)\right\}
$$

To exploit this we need a formula for the generalized parallel volume $V_{d}\left(N_{0}+r B^{*}\right)$. We start with the Euclidean case $B=B^{d}$, the general case will be treated later. For $\varphi \in \mathbf{N}$ we define

$$
\varphi^{(0)}:=\varphi \backslash\{0\}
$$

A straightforward argument yields for all $\varphi \in \mathbf{N}$ and all $r>0$ the formula

$$
\begin{gathered}
V_{d}\left(\varphi+r B^{d}\right)=\int_{0}^{r} \int_{S^{d-1}} \int_{\mathbb{R}^{d}} 1\left\{B^{d}(t u, t) \cap(\varphi-x)^{(0)}=\emptyset\right\} \\
\times t^{d-1} \varphi(d x) \mathcal{H}^{d-1}(d u) d t
\end{gathered}
$$


where $S^{d-1}$ is the unit sphere in $\mathbb{R}^{d}$ and $B^{d}(x, r)$ is the Euclidean ball with radius $r$ and center at $x$. Formally, this is a very special case of the generalized Steiner formula in [21], Theorem 3.1. Inserting (24) into 23, we obtain that

$$
1-H_{s}(r)=\exp \left\{-\int_{0}^{r} \lambda_{s}(t) d t\right\}
$$

where

$$
\lambda_{s}(t):=\gamma_{p} t^{d-1} \int_{S^{d-1}} \mathbb{E}\left[\int_{\mathbb{R}^{d}} \mathbf{1}\left\{\left(N_{0}-x\right)^{(0)} \cap B^{d}(t u, t)=\emptyset\right\} N_{0}(d x)\right] \mathcal{H}^{d-1}(d u) .
$$

In case $N_{0}=\{0\}$ we have $\Phi=\Phi_{p}$ and

$$
\lambda_{s}(t)=\gamma d b_{d} t^{d-1}
$$

in accordance with (12). (Note that here $\gamma_{c}=1$.) Adapting the terminology used in reliability theory to our situation, we call $\lambda_{s}$ the empty space hazard of $\Phi$ (see [7]). Loosely speaking, we have

$$
\mathbb{P}(d(0, \Phi) \in(t, t+h] \mid d(0, \Phi) \geq t) \approx \lambda_{s}(t) h
$$

for small $h>0$. Formula (25) has been proved in [20]. Additional formulae for the spherical contact distribution functions of some special Poisson cluster processes can be found in [35] and [44].

\subsection{Gauss-Poisson Processes}

In this subsection we assume that the cluster $N_{0}$ has at most 2 points, hence $\eta:=$ $N_{0}\left(\mathbb{R}^{d}\right) \leq 2$. Then we can write

$$
N_{0}= \begin{cases}\emptyset & \text { if } \eta=0 \\ \left\{Y_{0}\right\} & \text { if } \eta=1 \\ \left\{Y_{1}, Y_{2}\right\} & \text { if } \eta=2\end{cases}
$$

where $Y_{0}, Y_{1}, Y_{2}$ are random vectors in $\mathbb{R}^{d}$ with $Y_{1} \neq Y_{2}$. If $Y_{0}=Y_{1}=0$ and $\eta$ is independent of $Y_{2}$, then $\Phi$ is called Gauss-Poisson process. We have

$$
\begin{aligned}
& \mathbb{E}\left[\int_{\mathbb{R}^{d}} \mathbf{1}\left\{\left(N_{0}-x\right)^{(0)} \cap B^{d}(t u, t)=\emptyset\right\} N_{0}(d x)\right]=\mathbb{P}(\eta=1) \\
& \quad+\mathbb{P}(\eta=2) \mathbb{P}\left(Y_{1}-Y_{2} \notin B^{d}(t u, t) \mid \eta=2\right) \\
& \quad+\mathbb{P}(\eta=2) \mathbb{P}\left(Y_{2}-Y_{1} \notin B^{d}(t u, t) \mid \eta=2\right)
\end{aligned}
$$

(if $\mathbb{P}(\eta=2)>0$ ), and therefore (26) simplifies to

$$
\begin{aligned}
t^{1-d} \lambda_{s}(t)= & \gamma_{p} d b_{d} \mathbb{P}(\eta=1) \\
& +2 \mathbb{P}(\eta=2) \gamma_{p} \int_{S^{d-1}} \mathbb{P}\left(Y_{1}-Y_{2} \notin B^{d}(t u, t) \mid \eta=2\right) \mathcal{H}^{d-1}(d u)
\end{aligned}
$$

(see also Example 5.2 in [20]). 


\subsection{Neyman-Scott Processes}

We now consider the case that the typical cluster is given by

$$
N_{0}= \begin{cases}\emptyset & \text { if } \eta=0 \\ \left\{Y_{i, n}: i=1, \ldots, n\right\} & \text { if } \eta=n \geq 1\end{cases}
$$

where the random cluster size $\eta$ is a random element of $\mathbb{N}_{0}$, and the $Y_{i, n}, n \in \mathbb{N}$, $i=1, \ldots, n$, are independent and identically distributed random elements of $\mathbb{R}^{d}$ that are independent of $\eta$. In this case $\gamma_{c}=\mathbb{E} \eta$ which we have assumed to be finite. The Poisson cluster process $\Phi$ is then called a Neyman-Scott process (see also $\S 16.2$ in [44]). Let $\mathbb{V}$ denote the distribution of $Y_{1,1}$. We have

$$
\begin{aligned}
\mathbb{E} & {\left[\int_{\mathbb{R}^{d}} \mathbf{1}\left\{\left(N_{0}-x\right)^{(0)} \cap B^{d}(t u, t)=\emptyset\right\} N_{0}(d x)\right] } \\
& =\mathbb{P}(\eta=1)+\sum_{n=2}^{\infty} n \mathbb{P}(\eta=n) \mathbb{P}\left(Y_{i, n}-Y_{1, n} \notin B^{d}(t u, t), i=2, \ldots, n\right) \\
& =\mathbb{P}(\eta=1)+\sum_{n=2}^{\infty} n \mathbb{P}(\eta=n) \int_{\mathbb{R}^{d}} \mathbb{P}\left(Y_{1,1}-x \notin B^{d}(t u, t)\right)^{n-1} \mathbb{V}(d x) \\
& =\int_{\mathbb{R}^{d}} g^{\prime}\left(\mathbb{P}\left(Y_{1,1}-x \notin B^{d}(t u, t)\right)\right) \mathbb{V}(d x),
\end{aligned}
$$

where $g^{\prime}$ is the derivative of the probability generating function $g$ of $\eta$. Hence we conclude from (26) that

$$
\lambda_{s}(t)=\gamma_{p} t^{d-1} \int_{S^{d-1}} \int_{\mathbb{R}^{d}} g^{\prime}\left(\mathbb{P}\left(Y_{1,1}-x \notin B^{d}(t u, t)\right)\right) \mathbb{V}(d x) \mathcal{H}^{d-1}(d u),
$$

correcting a statement in Example 5.1 of [20].

\subsection{Asymptotic Behaviour of the Empty Space Hazard}

Let $\Phi$ be a general Poisson cluster process as in Sects. 6.1 and 6.2. Formula 26 gives the empty space hazard $\lambda_{s}$ of $\Phi$ in terms of the parent intensity $\gamma_{p}$ and the distribution of the typical cluster $N_{0}$. Although informative, this formula is still rather complicated. It is therefore instructive to compare $\lambda_{s}$ as given by (26) with the right side of (27). From monotone convergence

$$
\lim _{t \rightarrow 0} t^{1-d} \lambda_{s}(t)=\gamma d b_{d}
$$

i.e. for small values of $t$ the empty space hazard of a Poisson cluster process behaves approximately like the empty space hazard of a Poisson process with the same intensity. As $t \rightarrow \infty$ we have $B^{d}(t u, t) \uparrow\{0\} \cup\left\{x \in \mathbb{R}^{d}:\langle x, u\rangle>0\right\}$ for each $u \in S^{d-1}$ and it easily follows that 


$$
\lim _{t \rightarrow \infty} t^{1-d} \lambda_{s}(t)=\gamma_{p} \int_{S^{d-1}} \mathbb{E}\left[\int_{\mathbb{R}^{d}} \mathbf{1}\left\{\left(N_{0}-x\right)^{(0)} \cap H_{u}^{+}=\emptyset\right\} N_{0}(d x)\right] \mathcal{H}^{d-1}(d u),
$$

where $H_{u}^{+}:=\left\{x \in \mathbb{R}^{d}:\langle x, u\rangle \geq 0\right\}$. It is an interesting fact that the convergence in (31) and (32) is monotone, i.e. that $t^{1-d} \lambda_{s}(t)$ is monotone decreasing in $t$. The righthand side of (32) can be considerably simplified. Splitting the expectation according to the events $N_{0}\left(\mathbb{R}^{d}\right)=n, n \in \mathbb{N}$, we have to determine the integrals

$$
\int_{S^{d-1}}\left[\sum_{k=1}^{n} \mathbb{P}\left(\left\langle Y_{j}-Y_{k}, u\right\rangle \leq 0 \text { for all } j \neq k, N_{0}\left(\mathbb{R}^{d}\right)=n\right)\right] \mathcal{H}^{d-1}(d u),
$$

for all $n \geq 2$, where $Y_{1}, \ldots, Y_{n}$ are pairwise distinct (random) vectors in $\mathbb{R}^{d}$. Fixing $u \in S^{d-1}$ and assuming without loss of generality that $\left\langle Y_{j}-Y_{k}, u\right\rangle \neq 0$ for $j \neq k$, it can be shown by induction that the above sum in brackets is just $\mathbb{P}\left(N_{0}\left(\mathbb{R}^{d}\right)=n\right)$. Hence we obtain from (32) that

$$
\lim _{t \rightarrow \infty} t^{1-d} \lambda_{s}(t)=\mathbb{P}\left(N_{0} \neq \emptyset\right) \gamma_{p} d b_{d}
$$

irrespective of any specific assumptions on the typical cluster $N_{0}$. This is the same asymptotics as that of the Poisson process $\left\{x \in \Phi_{p}: N_{x} \neq \emptyset\right\}$. The latter has intensity $\mathbb{P}\left(N_{0} \neq \emptyset\right) \gamma_{p}$. This means in a sense, that different cluster points cannot be distinguished from a very far distance.

\section{Local Geometric Concepts}

\subsection{Support Measures}

The Minkowski functionals are important characteristics of convex bodies and of sets in the convex ring (polyconvex bodies). For convex bodies, they can be obtained as coefficients of a Steiner formula, for polyconvex bodies they are determined by their additivity properties. We now describe how these functionals can be localized to measurevalued functionals which appear as coefficient measures of a local Steiner formula. We have already seen in the second part of Sect.5that it is important to gain local directional information about random $\mathcal{S}^{d}$-sets. The (Euclidean) support measures (or generalized curvature measures) $\Theta_{i}(K ; \cdot), i=0, \ldots, d-1$, of a convex body $K \in \mathcal{K}^{d}$, which we introduce now, are essential geometric tools useful to quantify this local information. The support measures are finite measures on $\mathbb{R}^{d} \times S^{d-1}$ which can be obtained as coefficients of a local Steiner formula, and whose definition is based on the underlying Euclidean structure and therefore on the geometric properties of the Euclidean unit ball. These measures, and in particular the surface area measures $S_{i}(K ; \cdot):=\Theta_{i}\left(K ; \mathbb{R}^{d} \times \cdot\right)$, $i=0, \ldots, d-1$, have been used in the literature since quite a while. (Recall that we already introduced the surface area measure $S_{d-1}(K ; \cdot)$ in Sect. 5.) Of more recent vintage are (relative) support measures which are defined with respect to a more general convex gauge body $B$ (with $0 \in B$ ) which replaces the Euclidean unit ball. 
In order to be consistent with the literature, we first set $d_{B}(K, x):=d_{B^{*}}(x, K)$ and $u_{B}(K, x):=-u_{B^{*}}(x, K)$ for $x \in \mathbb{R}^{d} \backslash K$. Furthermore, we assume that $K$ and $B$ are in general relative position, which means that $K$ and $B$ do not contain parallel segments in parallel and equally oriented support (hyper)planes. A sufficient condition is that $K$ or $B$ is strictly convex, but the condition is also satisfied for an arbitrary convex body $K$ and almost all rotational images of an arbitrary convex body $B$. For convex bodies $K$ and $B$ in general relative position (with $0 \in B$ ), there are finite measures $\Theta_{0}(K ; B ; \cdot), \ldots, \Theta_{d-1}(K ; B ; \cdot)$ on $\mathbb{R}^{d} \times \partial B$ which satisfy the local Steiner formula

$$
\begin{aligned}
V_{d}(\{x \in(K+r B) \backslash & \left.\left.\backslash K:\left(x-d_{B}(K, x) u_{B}(K, x), u_{B}(K, x)\right) \in \cdot\right\}\right) \\
& =\frac{1}{d} \sum_{j=0}^{d-1} r^{d-j}\left(\begin{array}{l}
d \\
j
\end{array}\right) \Theta_{j}(K ; B ; \cdot),
\end{aligned}
$$

for all $r \geq 0$. The crucial point here is that the local parallel volume on the left side is a polynomial in the distance parameter $r$, and the coefficients of this polynomial are measures. These measures are called relative support measures of $K$ with respect to $B$; for further details see [14, 15, 18, 38]. Note that $\Theta_{j}(K ; B ; \cdot)$ is concentrated on $\partial K \times \partial B$ and that

$$
\Theta_{j}(K ; \cdot):=\Theta_{j}\left(K ; B^{d} ; \cdot\right)
$$

just defines the Euclidean support measures. The relative surface area measures $S_{j}(K ; B ; \cdot):=\Theta_{j}\left(K ; B ; \mathbb{R}^{d} \times \cdot\right)$ and the ordinary surface area measures $S_{j}(K ; \cdot)$ arise as image measures of (relative) support measures. Especially, we get

$$
\Theta_{j}(K ; B ; \partial K \times \partial B)=d V(K[j], B[d-j])
$$

and, in particular,

$$
\Theta_{j}\left(K ; \mathbb{R}^{d} \times S^{d-1}\right)=\frac{d b_{d-j}}{\left(\begin{array}{l}
d \\
j
\end{array}\right)} V_{j}(K),
$$

for $j=0, \ldots d-1$.

\subsection{Smooth Convex Bodies}

For sufficiently smooth convex bodies, the Euclidean surface area measures can be described in terms of basic curvature functions. Let $h_{K}(u):=\max \{\langle x, u\rangle: x \in K\}$, $u \in \mathbb{R}^{d}$, denote the support function of $K \in \mathcal{K}^{d}$ at $u$. We say that $K$ is of class $C_{+}^{2}$ if $\partial K$ is a hypersurface of class $C^{2}$ with everywhere positive Gauss curvature. Now let $K \in \mathcal{K}^{d}$ be of class $C_{+}^{2}$. This assumption implies that $h_{K}$ is of class $C^{2}$ and $K$ is strictly convex. The gradient $\nabla h_{K}(u)$ is the unique boundary point $\tau(K, u)$ of $K$ with exterior unit normal vector $u$. The principal radii of curvature of $K$ in (direction) $u$ are the reciprocals of the principal curvatures of $K$ at $\tau(K, u)$. Writing $s_{j}(K, u)$, $j \in\{1, \ldots, d-1\}$, for the $j$-th normalized elementary symmetric function of the principal radii of curvature of $K$ in $u$ and setting $s_{0}(K, u):=1$, for all $u \in S^{d-1}$, we get for any $K \in \mathcal{K}^{d}$ of class $C_{+}^{2}$ that

$$
S_{j}(K ; \cdot)=\int_{S^{d-1}} \mathbf{1}\{u \in \cdot\} s_{j}(K, u) \mathcal{H}^{d-1}(d u) .
$$


This equation provides a relationship between the coefficients of the local Steiner formula for the convex body $K$ and second order (local) geometric information on the bounding hypersurface $\partial K$. For more details we refer to [37].

\subsection{Additive Extensions of Support Measures}

An important property which the relative support measures share with the Minkowski functionals is additivity. As a consequence these measures can be extended as additive, signed, measure-valued functionals to the (extended) convex ring, and we use the same notation for these extensions. Later we will make use of $\Theta_{d-1}(K ; B ; \cdot), K \in \mathcal{S}^{d}$. Now we provide some information on these measures, but we have to refer to [14] for the details. We assume that the gauge body $B$ is strictly convex and contains the origin in its interior. Then the measure $\Theta_{d-1}(K ; B ; \cdot)$ is non-negative and concentrated on the relative (generalized) normal bundle $\mathcal{N}_{B}(K)$ of $K$ which is defined by

$$
\mathcal{N}_{B}(K):=\left\{\left(x-d_{B}(K, x) u_{B}(K, x), u_{B}(K, x)\right): x \notin K \cup \operatorname{exo}_{B^{*}}(K)\right\} .
$$

Furthermore, it can be expressed in terms of the Euclidean support measure $\Theta_{d-1}(K ; \cdot)$ and the support function $h_{B}$ of $B$ :

$$
\Theta_{d-1}(K ; B ; \cdot)=\int_{\mathcal{N}_{B^{d}}(K)} \mathbf{1}\left\{\left(x, \nabla h_{B}(u)\right) \in \cdot\right\} h_{B}(u) \Theta_{d-1}(K ; d(x, u)) .
$$

Assume that $K$ is regular, i.e. coincides with the closure of its interior. Then $\Theta_{d-1}(K ; \cdot)$ is just an integral over the boundary of $K$ :

$$
\Theta_{d-1}(K ; \cdot)=\int_{\partial K} \mathbf{1}\{(x, \nu(K, x)) \in \cdot\} \mathcal{H}^{d-1}(d x) .
$$

Here $\nu(K, x)$ denotes the outer normal of $K$ at the boundary point $x$, which is uniquely defined for $\mathcal{H}^{d-1}$-a.e. $x \in \partial K$.

\section{Poisson Cluster Models}

In this section we consider generalized contact distribution functions of grain models which are constructed from stationary Poisson cluster processes with independent marking. Especially, we describe formulas for the local contact distributions of Poisson cluster processes and Boolean models.

\subsection{Definition of a Poisson Cluster Model}

We continue the discussion of Poisson cluster processes by considering the grain model $Z$ (with convex grains) defined by (4), where $\Phi$ is a stationary Poisson cluster process (compare Sect.6) and $Z_{1}, Z_{2}, \ldots$ is a sequence of independent and identically distributed random convex bodies which is independent of $\Phi=\left\{\xi_{i}: i=1,2, \ldots\right\}$. Again, we write $\mathbb{Q}$ for the common distribution of the grains and $Z_{0}$ for the typical grain, i.e. a random 
convex body with distribution $\mathbb{Q}$. From Sect. 3 we recall the standard assumption (6) which ensures that $\left\{Z_{i}+\xi_{i}: i=1,2, \ldots\right\}$ is a locally finite particle process. It can be easily shown (compare [8]) that the capacity functional of $Z$ is given by

$$
T_{Z}(C)=1-\exp \left\{-\gamma_{p} \int\left(1-\mathbb{E}\left[\prod_{x \in N_{0}+y} \mathbb{P}\left(Z_{0} \cap(C-x)=\emptyset\right)\right]\right) d y\right\}
$$

for all $C \in \mathcal{C}^{d}$. On the other hand, the random closed set $Z$ thus obtained can also be interpreted as a stationary Boolean model with typical (compact, but not necessarily convex) grain

$$
\bigcup_{x \in N_{0}}(Z(x)+x)
$$

where $\left\{Z(x): x \in N_{0}\right\}$ is a family of random convex bodies that are conditionally independent given $N_{0}$ and where the conditional distribution of $Z(x)$ given $N_{0}$ is $\mathbb{Q}$. In order to check this, one shows that the capacity functional of such a Boolean model is equal to the functional 36 . The capacity functional of $Z$ can be used to derive formulas for the contact distribution functions of $Z$. However, in order to include contact directions, we have to pursue a different approach.

The reduced second moment measure of $N_{0}$ is the measure on $\mathbb{R}^{d}$ defined by

$$
\alpha_{0}:=\mathbb{E}\left[\sum_{\substack{x, y \in N_{0} \\ x \neq y}} 1\{x-y \in \cdot\}\right]
$$

Throughout the section we will assume that this measure is absolutely continuous.

\subsection{The Spherical Contact Distribution of a Poisson Cluster Model}

In this subsection we study the generalized spherical contact distribution function defined by (11) with $B=B^{d}$, that is

$$
H_{s}(r, C):=\mathbb{P}(d(0, Z) \leq r, u(0, Z) \in C \mid 0 \notin Z),
$$

where $r \geq 0$ and $C \subset S^{d-1}$ is a Borel set. First we define the means of the support measures with respect to the distribution $\mathbb{Q}$ of the typical grain (reflected at the origin),

$$
\bar{\Theta}_{j}^{*}:=\int \Theta_{j}\left(K^{*} ; \cdot\right) \mathbb{Q}(d K) .
$$

By our integrability assumption on $\mathbb{Q}$, we thus obtain finite measures on $\mathbb{R}^{d} \times S^{d-1}$. For given $\psi=\sum_{n=1}^{m} \delta_{x_{n}}$, we let $\Gamma(\psi, \cdot)$ denote the distribution of the random closed set $\cup_{n=1}^{m}\left(Z_{n}+x_{n}\right)$, where $Z_{1}, \ldots, Z_{m}$ are independent with distribution $\mathbb{Q}$. We also set $\Gamma(\emptyset, \cdot):=\delta_{\emptyset}$. Finally, we define

$$
\mu_{0}:=\mathbb{E}\left[\int_{\mathbb{R}^{d}} \int_{\mathcal{F}^{d}} \mathbf{1}\{A \in \cdot\} \Gamma\left(\left(N_{0}-y\right)^{(0)}, d A\right) N_{0}(d y)\right],
$$


which is a finite measure on $\mathcal{F}^{d}$. Then

$$
\begin{aligned}
H_{s}(r, C)=\gamma_{p} \sum_{j=0}^{d-1}\left(\begin{array}{c}
d-1 \\
j
\end{array}\right) \int_{0}^{r} \int_{\mathbb{R}^{d} \times C} \int_{\mathcal{F}^{d}}\left(1-H_{s}(t)\right) t^{d-1-j} \\
\times \mathbf{1}\{d(-x-t u, A)>t\} \mu_{0}(d A) \bar{\Theta}_{j}^{*}(d(x, u)) d t .
\end{aligned}
$$

This equation has been proved in [20] in the special case $C=\mathbb{R}^{d}$. For a general measurable set $C \subset \mathbb{R}^{d}$, 39) is a special case of Proposition 4.27 and Example 4.28 in [14]. Defining

$$
\begin{aligned}
\lambda_{s}(t, C):=\gamma_{p} \sum_{j=0}^{d-1} & \left(\begin{array}{c}
d-1 \\
j
\end{array}\right) t^{d-1-j} \int_{\mathbb{R}^{d} \times C} \int_{\mathcal{F}^{d}} \\
& \times \mathbf{1}\{d(-x-t u, A)>t\} \mu_{0}(d A) \bar{\Theta}_{j}^{*}(d(x, u)),
\end{aligned}
$$

we see that $H_{s}(\cdot, C)$ has the density $\lambda_{s}(t, C)\left(1-H_{s}(t)\right)$. The function $\lambda_{s}(\cdot, C)$ can be interpreted as a direction dependent empty space hazard, because

$$
\mathbb{P}(d(0, Z) \in(t, t+h], u(0, Z) \in C \mid d(0, Z) \geq t) \approx \lambda_{s}(t, C) h
$$

for small $h>0$. The choice $C=S^{d-1}$ leads to

$$
H_{s}(r)=1-\exp \left\{-\int_{0}^{r} \lambda_{s}(t) d t\right\}
$$

where $\lambda_{s}(t):=\lambda_{s}\left(t, S^{d-1}\right)$. If $\Phi$ is Poisson, then $Z$ is a Boolean model (with typical grain $Z_{0}$ ) and (41) simplifies to (16). Moreover, we have in this case

$$
\lambda_{s}(t, C)=\gamma \sum_{j=0}^{d-1} t^{d-j-1}\left(\begin{array}{c}
d-1 \\
j
\end{array}\right) \bar{S}_{j}^{*}(C),
$$

where

$$
\bar{S}_{j}^{*}:=\int \Theta_{j}\left(K^{*} ; \mathbb{R}^{d} \times \cdot\right) \mathbb{Q}(d K)=\bar{\Theta}_{j}^{*}\left(\mathbb{R}^{d} \times \cdot\right),
$$

is the mean of the $j$-th surface area measure $S_{j}\left(K^{*} ; \cdot\right)$ with respect to $\mathbb{Q}$ and $\gamma=\gamma_{p}$ since $\gamma_{c}=1$.

As $t \rightarrow 0$, the inner integral of 40 tends to

$$
\int_{\mathcal{F}^{d}} \mathbf{1}\left\{x \notin A^{*}\right\} \mu_{0}(d A) .
$$

Furthermore,

$$
\lim _{t \rightarrow \infty} t^{1-d} \lambda_{s}(t, C)=\gamma_{p} \int_{\mathbb{R}^{d} \times C} \int_{\mathcal{F}^{d}} 1\left\{\left(H_{u, x} \cup\{x\}\right) \cap A^{*}=\emptyset\right\} \mu_{0}(d A) \bar{\Theta}_{0}^{*}(d(x, u)),
$$

where $H_{u, x}:=\left\{x+y: y \in \mathbb{R}^{d},\langle y, u\rangle>0\right\}$. 


\subsection{Examples}

We now discuss cluster models based on the two special cluster processes introduced in Sects. 6.3 and 6.4. First we consider the typical cluster given by (28). In order to guarantee the absolute continuity of the measure (38) we assume that the conditional distribution of $Y_{1}-Y_{2}$ given that $\eta=2$ is absolutely continuous. Equation (40) takes the form

$$
\begin{aligned}
\lambda_{s}(t, C) & =\gamma_{p} \mathbb{P}(\eta=1) \sum_{j=0}^{d-1}\left(\begin{array}{c}
d-1 \\
j
\end{array}\right) t^{d-1-j} \bar{S}_{j}^{*}(C) \\
+ & \gamma_{p} \mathbb{P}(\eta=2) \sum_{j=0}^{d-1}\left(\begin{array}{c}
d-1 \\
j
\end{array}\right) t^{d-1-j} \\
& \times \int_{\mathbb{R}^{d} \times C}\left\{\mathbb{P}\left(\left(Z_{1}+Y_{2}-Y_{1}\right) \cap B^{d}(-x-t u, t)=\emptyset \mid \eta=2\right)\right. \\
& \left.+\mathbb{P}\left(\left(Z_{2}+Y_{1}-Y_{2}\right) \cap B^{d}(-x-t u, t)=\emptyset \mid \eta=2\right)\right\} \bar{\Theta}_{j}^{*}(d(x, u)),
\end{aligned}
$$

where $Z_{1}, Z_{2}$ have distribution $\mathbb{Q}$ and $Y_{1}-Y_{2}, Z_{i}$ are independent for $i=1,2$.

Next we consider a Neyman-Scott process $\Phi$ as defined at (29) and assume that the distribution of $Y_{1,2}-Y_{2,2}$ is absolutely continuous. The measure (38) is then absolutely continuous and we obtain similarly as above

$$
\begin{aligned}
\lambda_{s}(t, C)=\gamma_{p} \sum_{j=0}^{d-1}\left(\begin{array}{c}
d-1 \\
j
\end{array}\right) t^{d-j-1} \int_{\mathbb{R}^{d} \times C} \int_{\mathbb{R}^{d}} & \\
& \times g^{\prime}\left(\mathbb{P}\left(\left(Z_{0}+Y_{0}-y\right) \cap B^{d}(-x-t u, t)=\emptyset\right)\right) \mathbb{V}(d y) \bar{\Theta}_{j}^{*}(d(x, u)),
\end{aligned}
$$

where $Y_{0}$ has the distribution $\mathbb{V}$ of the $Y_{i, n}$ and is independent of the typical grain $Z_{0}$. This corrects a statement in Example 5.3 of [20].

Formulas (44) and (45) can be used to compute $\lambda_{s}(t)=\lambda_{s}\left(t, S^{d-1}\right)$ via MonteCarlo integration. Clearly this requires more specific assumptions on the typical grain. A convenient and popular choice is a spherical grain $Z_{0}=\tau B^{d}$, where $\tau$ is a positive random variable. In [20], it has been shown (for $d=3$ ) that the empty space hazard might be an appropriate tool to reveal clustering phenomena. However, if the typical cluster $N_{0}$ contains at most two points (as in the Gauss-Poisson case, for instance) then the empty space hazard can very much resemble that of a Boolean model. If, on the other hand, $N_{0}$ contains three or even more points, then the empty space between the grains of the cluster cannot be neglected anymore and a quadratic function cannot be fitted to the rate in a satisfactory manner.

\subsection{General Gauge Bodies}

Now we turn to the generalized contact distribution of $Z$ with respect to a general gauge body $B$. Since for our analysis we have to use relative support measures with respect to $B$, we now make the further asumption that $B^{*}$ and the typical grain $Z_{0}$ are almost 
surely in general relative position. If $B$ is strictly convex, or if $\mathbb{Q}$ is concentrated on the set of strictly convex bodies, then this assumption is automatically satisfied. Extending a previous definition, we set

$$
\bar{\Theta}_{j}^{*}(B ; \cdot):=\int \Theta_{j}\left(K^{*} ; B ; \cdot\right) \mathbb{Q}(d K), \quad j=0, \ldots, d-1,
$$

and obtain finite measures on $\mathbb{R}^{d} \times \partial B$. As a generalization of [39], we have

$$
\begin{aligned}
H_{B}(r, C)=\gamma_{p} \sum_{j=0}^{d-1}\left(\begin{array}{c}
d-1 \\
j
\end{array}\right) \int_{0}^{r} \int_{\mathbb{R}^{d} \times C} \int_{\mathcal{F}^{d}}\left(1-H_{B}(t)\right) t^{d-1-j} \\
\times \mathbf{1}\left\{d_{B}(-x-t u, A)>t\right\} \mu_{0}(d A) \bar{\Theta}_{j}^{*}(B ; d(x, u)) d t
\end{aligned}
$$

for all $r \geq 0$ and Borel sets $C \subset \partial B$. For smooth $B$ this has been proved in [14]. The present more general result can be obtained by combining a special case of Theorem 5.1 in [15] with Example 4.28 in [14]. In Sect.10, we will describe a further extension to instationary models.

The special case $Z_{0}=\{0\}$ of (39) deserves some discussion. In that case, $Z=\Phi$ and

$$
\begin{aligned}
H_{B}(r, C)=\gamma_{p} & \int_{0}^{r} \int_{C}\left(1-H_{B}(t)\right) t^{d-1} \\
& \times \mathbb{E}\left[\int_{\mathbb{R}^{d}} \mathbf{1}\left\{d_{B}\left(-t u,\left(N_{0}-y\right)^{(0)}\right)>t\right\} N_{0}(d y)\right] \Theta_{B}(d u) d t,
\end{aligned}
$$

where

$$
\Theta_{B}:=d \int_{B} \mathbf{1}\left\{x / d_{B}(0, x) \in \cdot\right\} d x .
$$

This is just the measure $\Theta_{0}(\{0\} ; B ;\{0\} \times \cdot)$ with total mass $d \cdot V_{d}(B)$. If $B$ is lower dimensional, then $\Theta_{B}$ is the zero measure and the right side of (47) vanishes identically, in accordance with $\mathbb{P}\left(d_{B}(0, \Phi)<\infty\right)=0$. In case $B=B^{d}$ we may use polar coordinates to see that $\Theta_{B^{d}}$ is the Hausdorff measure $\mathcal{H}^{d-1}$ on $S^{d-1}$. More generally, for any $B \in \mathcal{K}^{d}$ with 0 as an interior point one has

$$
\Theta_{B}=\int_{\partial B} 1\{u \in \cdot\} h_{B}(\nu(B, u)) \mathcal{H}^{d-1}(d u) .
$$

Suppose that the expectation

$$
\mathbb{E}\left[\int_{\mathbb{R}^{d}} \mathbf{1}\left\{d_{B}\left(-t u,\left(N_{0}-y\right)^{(0)}\right)>t\right\} N_{0}(d y)\right]
$$

does not depend on $u \in \partial B$ for all $t>0$. This is the case if the cluster distribution is isotropic, i.e. invariant under rotations around the origin. Equation (47) then implies the independence of $d_{B}(0, \Phi)$ and $u_{B}(0, \Phi)$. In the Poisson case (where isotropy is fulfilled automatically), 


$$
H_{B}(r, C)=\gamma \Theta_{B}(C) \int_{0}^{r}\left(1-H_{B}(t)\right) t^{d-1} d t,
$$

or

$$
H_{B}(r, C)=\left(d V_{d}(B)\right)^{-1} \Theta_{B}(C)\left(1-\exp \left[-\gamma r^{d} V_{d}(B)\right]\right) .
$$

Other special cases of (46) and (47) can be discussed as before.

\section{General Stationary Random Sets}

In this section, we consider a general stationary random $\mathcal{S}^{d}$-set $Z$ and discuss the information contained in the contact distribution function $H_{B}$ of $Z$, where $B$ is a general gauge body. Even under these weak assumptions, we can deduce some regularity properties of $H_{B}$.

\subsection{The General Form of Direction Dependent Contact Distributions}

As explained in Sect. 3 we may represent $Z$ as a stationary grain model $Z=\bigcup_{n \in \mathbb{N}}\left(Z_{n}+\right.$ $\left.\xi_{n}\right)$ with underlying stationary marked point process $\Psi=\left\{\left(\xi_{n}, Z_{n}\right): n \in \mathbb{N}\right\}$ on $\mathbb{R}^{d} \times \mathcal{K}^{d}$. Since $\Psi$ is stationary, the intensity measure $\tilde{\Lambda}$ of $\Psi$ can be decomposed in the form $\tilde{\Lambda}=\gamma V_{d} \otimes \mathbb{Q}$, where $\gamma$ is the intensity and $\mathbb{Q}$ is the grain distribution of $\Psi$ (compare Sect. 3.3). We assume that the structuring element $B$ and $K^{*}$ are in general relative position for $\mathbb{Q}$-almost all $K$ and that $\Psi$ satisfies a weak regularity condition (for details, see [14, 15]). Then we obtain

$$
\begin{aligned}
& (1-p) H_{B}(r, C)=\gamma \sum_{j=0}^{d-1}\left(\begin{array}{c}
d-1 \\
j
\end{array}\right) \int_{0}^{r} \int_{\mathcal{K}^{d}} \int_{\mathbb{R}^{d} \times C} t^{d-1-j} \\
& \times \mathbb{P}^{(0, K)}\left(\left\{\psi: d_{B}(-y-t u, Z(\psi, K))>t\right\}\right) \Theta_{j}\left(K^{*} ; B ; d(y, b)\right) \mathbb{Q}(d K) d t
\end{aligned}
$$

where

$$
Z(\psi, K):=\bigcup_{(x, L) \in \psi \backslash\{(0, K)\}}(L+x),
$$

and where the probability measures $\mathbb{P}^{(0, K)}, K \in \mathcal{K}^{d}$, are the Palm distributions of $\Psi$. The latter can be interpreted as conditional distributions of $\Psi$ given that $(0, K) \in \Psi$; see $\S 3.4$ and $\S 4.3$ in [39]. In [15], a more general result was deduced without the assumption of stationarity. The statement of such a result requires the general Palm distributions $\mathbb{P}^{(x, K)},(x, K) \in \mathbb{R}^{d} \times \mathcal{K}^{d}$, of $\Psi$. Relation (50) can be deduced either directly or from the more general result by means of

$$
\mathbb{P}^{(x, K)}=\int \mathbf{1}\{\psi+x \in \cdot\} \mathbb{P}^{(0, K)}(d \psi)
$$

which holds for $\tilde{\Lambda}$-a.e. $(x, K) \in \mathbb{R}^{d} \times \mathcal{K}^{d}$, where $\psi+x:=\{(y+x, K):(y, K) \in \psi\}$. From equation (50) we can deduce several further consequences. A first observation is that (50) implies that $H_{B}(\cdot, C)$ is absolutely continuous and a version of the density is 
given explicitly. In particular, we see that the (right) derivative of $H_{B}(\cdot, C)$ at 0 exists and can be expressed in the form

$$
\begin{aligned}
(1-p) H_{B}^{\prime}(0, C)=\gamma \int_{\mathcal{K}^{d}} & \int_{\mathbb{R}^{d} \times C} \mathbb{P}^{(0, K)}(\{\psi:-y \notin Z(\psi, K)\}) \\
& \times \Theta_{d-1}\left(K^{*} ; B ; d(y, b)\right) \mathbb{Q}(d K) d t .
\end{aligned}
$$

A related version of such a result is stated as Proposition 5.4 in [14], the additional assumptions on $B$ (such as smoothness), which are adopted in [14], can be removed. The absolute continuity of $H_{B}$ was already shown in [2] and [7] for rather general random closed sets, though in a less explicit and in a nonlocal form.

\subsection{First Derivatives and Surface Intensities}

In the remaining part of this section we assume that $B$ is strictly convex and 0 is in the interior of $B$. Using the mean of the support measure $\Theta_{d-1}\left(Z ; B^{*} ; \cdot\right)$ (see Sect.7.3) the derivatives $H_{B}^{\prime}(0, C), C \subset \partial B$, can be expressed as

$$
(1-p) H_{B}^{\prime}(0, C)=\mathbb{E} \Theta_{d-1}\left(Z ; B^{*} ;[0,1]^{d} \times C^{*}\right) .
$$

This formula holds without any reference to a marked point process $\Psi$ from which $Z$ may be derived. The right-hand side of this formula can be interpreted as a direction dependent (relative) surface density of $Z$. Since $\mathbb{E} \Theta_{d-1}\left(Z ; B^{*} ; \cdot \times \partial B^{*}\right)$ is a locally finite (by assumption), translation invariant, non-negative measure, it follows that

$$
\mathbb{E} \Theta_{d-1}\left(Z ; B^{*} ; \cdot \times \partial B^{*}\right)=\lambda_{d-1 ; B} V_{d}
$$

where the constant $\lambda_{d-1 ; B}:=\mathbb{E} \Theta_{d-1}\left(Z ; B^{*} ;[0,1]^{d} \times \partial B^{*}\right)$ is the (relative) surface density of $Z$. (By assumption this number is finite.) Hence

$$
H_{B}^{\prime}(0)=\lambda_{d-1 ; B} /(1-p)
$$

In case $B=B^{d}$, the number $\lambda_{d-1 ; B}$ is just the classical surface density of $Z$.

Define $C^{d}:=[0,1]^{d}$ and let $\partial^{+} C^{d}$ denote the upper right boundary of $C^{d}$. Then, under a suitable integrability assumption,

$$
\begin{aligned}
\mathbb{E} \Theta_{d-1}\left(Z ; B^{*} ;[0,1]^{d} \times \cdot\right) & =\lim _{r \rightarrow \infty} \frac{\mathbb{E} S_{d-1}\left(Z \cap r W ; B^{*} ; \cdot\right)}{V_{d}(r W)} \\
& =\mathbb{E} S_{d-1}\left(Z \cap C^{d} ; B^{*} ; \cdot\right)-\mathbb{E} S_{d-1}\left(Z \cap \partial^{+} C^{d} ; B^{*} ; \cdot\right)
\end{aligned}
$$

for any $W \in \mathcal{K}^{d}$ with $V_{d}(W)>0$; moreover, if $Z$ is ergodic, then the expectation on the right side of (53) can be omitted and we obtain $\mathbb{P}$-a.s. equality. Furthermore, we have

$$
(1-p) H_{B}^{\prime}(0)=\int h\left(B^{*}, u\right) \mathbb{E} \Theta_{d-1}\left(Z ;[0,1]^{d} \times d u\right),
$$

which is a consequence of (35) and (51) that will be used below. All these assertions follow from results which were obtained in a general non-stationary setting in [14], 
Sects. 4 and 5, and from [13], Sect. 3. Finally, we remark that if $Z$ is assumed to be ergodic, then by a modification of the argument for Theorem 3.4 in [13] we get $\mathbb{P}$-a.s.

$$
(1-p) H_{B}^{\prime}(0)=\lim _{r \rightarrow \infty} \limsup _{\epsilon \downarrow 0} \frac{V_{d}\left(\left[\left(Z+\epsilon B^{*}\right) \backslash Z\right] \cap r W\right)}{\epsilon V_{d}(r W)} .
$$

Relation (55) remains true if 'lim sup' is replaced by 'lim inf'.

\subsection{Mean Normal Measure and Dilation Volumes of Stationary Boolean Models}

We now assume that $Z$ is a stationary Boolean model as defined in Sect.5.2 In this case relation (50) and Slivnyak's theorem for marked point processes (see Theorem 3.4.9 in [39]) directly yield that

$$
H_{B}(r, C)=\gamma \sum_{j=0}^{d-1}\left(\begin{array}{c}
d-1 \\
j
\end{array}\right) \bar{S}_{j}^{*}(B ; C) \int_{0}^{r} t^{d-1-j}\left(1-H_{B}(t)\right) d t
$$

where

$$
\bar{S}_{j}^{*}(B ; \cdot):=\bar{\Theta}_{j}^{*}\left(B ; \mathbb{R}^{d} \times \cdot\right)=\int \Theta_{j}\left(K^{*} ; B ; \mathbb{R}^{d} \times \cdot\right) \mathbb{Q}(d K)
$$

are the relative versions of the measures defined by (43). Relation (56) can also be obtained as a special case of equation (46). A more general result for a stationary Boolean model $Z$ with grains in $\mathcal{R}^{d}$ is contained in [13], Theorem 2.4. In particular, 56] implies that

$$
H_{B}^{\prime}(0)=\gamma \bar{S}_{d-1}^{*}(B ; \partial B)=\gamma \int h(B, u) \bar{S}_{d-1}^{*}(d u)
$$

which can also be deduced from equation (18). Moreover, since $Z+t B$ is a stationary Boolean model, for all $t \geq 0$, and therefore ergodic, we get $\mathbb{P}$-a.s.

$$
H_{B}(t)=\lim _{r \rightarrow \infty} \frac{V_{d}\left(\left[\left(Z+t B^{*}\right) \backslash Z\right] \cap r W\right)}{V_{d}(r W \backslash Z)},
$$

for any $t \geq 0$ and an arbitrary $W \in \mathcal{K}^{d}$ with $V_{d}(W)>0$. As in 19 we let $\bar{S}_{d-1}$ denote the mean normal measure of the typical grain of a stationary marked Poisson process $\Psi$ from which $Z$ is derived. Then, combining (57) and (58), we obtain for an arbitrary gauge body $B$ with $0 \in B$ that $\mathbb{P}$-a.s.

$$
\gamma \int h(B, u) \bar{S}_{d-1}(d u)=\lim _{\epsilon \downarrow 0} \lim _{r \rightarrow \infty} \frac{V_{d}([(Z+\epsilon B) \backslash Z] \cap r W)}{\epsilon V_{d}(r W \backslash Z)} .
$$

On the other hand, using (54), (55) and Theorem 4.1 in [49], we also get that $\mathbb{P}$-a.s.

$$
\gamma \int h(B, u) \bar{S}_{d-1}(d u)=\lim _{r \rightarrow \infty} \limsup _{\epsilon \downarrow 0} \frac{V_{d}([(Z+\epsilon B) \backslash Z] \cap r W)}{\epsilon V_{d}(r W \backslash Z)}
$$

for any $W \in \mathcal{K}^{d}$ with $V_{d}(W)>0$. Equation (60) holds for all convex bodies $B$ which contain the origin as a relative interior point; moreover, 'lim sup' can be replaced by 
'lim inf' in (60). Note that the limits on the right sides of (59) and 60) are taken in reversed order. Since the dilation volumes on the right sides of these equations can be determined in principle from observations of the Boolean model for all admissible convex bodies $B$, the oriented mean normal measure is determined. It is obvious now that these relations can be used to estimate the (unoriented) mean normal distribution of the underlying stationary Poisson particle process $X$, but the problems with the inversion of the Cosine transform which we mentioned in Sect.5] occur here as well.

\subsection{Second Derivatives}

Assume that $Z$ is a locally finite union of segments in $\mathbb{R}^{d}, d \geq 3$. (Such random sets $Z$ are also called segment processes, see e.g. [42].) Then the first derivative $H_{s}^{\prime}(0, C)$ vanishes, as follows from (52). However, for $d=3$, Corollary 5.2 in [14] implies that

$$
H_{s}^{\prime \prime}(0, C)=2 \pi L_{V} \mathcal{R}_{1}(C) .
$$

Here, $L_{V}$ is the length density of $Z$ and $\mathcal{R}_{1}(\cdot)$ is the (normalized) rose of directions of $Z$. Although not yet available in the literature, this result remains true for the union sets of stationary fibre processes in $\mathbb{R}^{3}$ (i.e. processes of curves with suitable regularity properties). Analogous results should hold for $k$-dimensional random sets $Z$, $k \in\{2, \ldots, d-2\}$.

For full-dimensional grains in $\mathbb{R}^{d}$ the situation becomes more complicated. In [23], a further step was made by considering the second derivative $H_{s}^{\prime \prime}(0)$ of the spherical contact distribution at 0 . As the authors show, for stationary grain models with smooth convex grains, and under some mild conditions on the size and relative position of the grains, the derivative $H_{s}^{\prime \prime}(0)$ exists (in the sense of Radon-Nikodym), but does not coincide with one of the standard functionals of $Z$. More precisely,

$$
H_{s}^{\prime \prime}(0)=\frac{2 \pi}{1-p}\left(\lambda_{d-2}^{+}-\mu_{d-2}\right),
$$

where $\lambda_{d-2}^{+}$is the intensity of the non-negative $(d-2)$-nd curvature measure of $Z$ (see [14 21]) and $\mu_{d-2}$ is a corresponding intensity of a measure which integrates the tangens of the 'outer' angle over all singular boundary points of $Z$ (see [23]). A heuristic discussion of the second derivative of the parallel volume $V_{2}\left(K+r B^{2}\right), K \in \mathcal{R}^{2}$, is given in [25].

\section{The Instationary Case}

\subsection{Introduction}

So far we mainly considered stationary point processes. If $\Phi$ is an arbitrary point process, then we can still define direction dependent contact distribution functions with respect to a structuring element $B$ by

$$
H_{B}(x, r, C):=\mathbb{P}\left(d_{B}(x, \Phi) \leq r, u_{B}(x, \Phi) \in C \mid x \notin \Phi\right),
$$


where $r \geq 0$ and $C \subset \partial B$ is a Borel set, provided that $\mathbb{P}(x \notin \Phi)>0$ and $u_{B}(x, \Phi)$ is well-defined $\mathbb{P}$-a.s. Notice that we now take into account the location $x \in \mathbb{R}^{d}$, i.e. we consider $x+t B, t>0$, as the growing set. In a similar way the contact distribution of an arbitrary random closed set is defined. In contrast to the stationary case, these functions depend on the choice of a reference point $x \in \mathbb{R}^{d}$. They can be studied by means of the family of Palm distributions of $\Phi$ (see [14]).

\subsection{The Instationary Poisson Process}

A general Poisson process $\Phi$ on $\mathbb{R}^{d}$ is a point process which has the same independence properties as a stationary Poisson process (i.e. for pairwise disjoint Borel sets $A_{1}, \ldots, A_{k} \subset \mathbb{R}^{d}$, the random variables $\Phi\left(A_{1}\right), \ldots, \Phi\left(A_{k}\right)$ are independent $)$ and which satisfies

$$
\mathbb{P}(\Phi(A)=m)=\frac{\Lambda(A)^{m}}{m !} \exp [-\Lambda(A)], \quad m=0,1, \ldots,
$$

for all Borel sets $A \subset \mathbb{R}^{d}$, where $\Lambda(A)=\mathbb{E} \Phi(A)$ is the mean number of points of $\Phi$ in the set $A$. $\Lambda$ is a measure on $\mathbb{R}^{d}$, which is called the intensity measure of $\Phi$. Since we have assumed from the very beginning that $\Phi(\{x\}) \leq 1$ for all $x \in \mathbb{R}^{d}$, it follows that the intensity measure $\Lambda$ is diffuse, i.e. that $\Lambda(\{x\})=0$ for all $x \in \mathbb{R}^{d}$. If the intensity measure $\Lambda$ of $\Phi$ is a multiple of Lebesgue measure, then $\Phi$ is a stationary Poisson process.

Assume now that $\Phi$ is a Poisson process with an absolutely continuous intensity measure $\Lambda$. Hence, for all Borel sets $A \subset \mathbb{R}^{d}$,

$$
\Lambda(A)=\int_{A} \gamma(x) d x
$$

where $\gamma: \mathbb{R}^{d} \rightarrow[0, \infty)$ is a measurable function, which may be called the intensity function of $\Phi$. This assumption ensures that $\mathbb{P}(x \in \Phi)=0$ and that $u_{B}(x, \Phi)$ is almost surely uniquely determined; moreover,

$$
H_{B}(x, r, C)=\int_{0}^{r} \int_{C}\left(1-H_{B}(x, t)\right) t^{d-1} \gamma(x+t u) \Theta_{B}(d u) d t
$$

where $H_{B}(x, t):=H_{B}(x, t, \partial B)$ and the measure $\Theta_{B}$ has been defined by [48.

\subsection{General Poisson Processes and Boolean Models}

A Poisson process can be defined on a general metric space $\mathbf{X}$. Given a diffuse and $\sigma$-finite measure $\Lambda$ on $\mathbf{X}$, the definition in the preceding subsection applies verbatim.

Consider a Poisson process $\Phi$ on $\mathbb{R}^{d}$ with intensity measure $\Lambda$ and let $\Psi=\left\{\left(\xi_{n}, Z_{n}\right)\right.$ : $n \in \mathbb{N}\}$ be an independent marking of $\Phi$ with mark distribution $\mathbb{Q}$ on $\mathcal{C}^{d}$. It is one of the nice and fundamental properties of the Poisson process (see e.g. [19, 39]) that $\Psi$ is a Poisson process on $\mathbb{R}^{d} \times \mathcal{C}^{d}$ with intensity measure $\tilde{\Lambda}=\Lambda \otimes \mathbb{Q}$. From the mapping theorem for Poisson processes (see [19]) we obtain that the particle process $X:=\left\{Z_{n}+\xi_{n}: n \in \mathbb{N}\right\}$ is a Poisson process on $\mathcal{C}^{d}$ (provided $X$ has a locally finite intensity measure). It is natural to generalize the definition in Sect.5.2 and to call 


$$
Z:=\bigcup_{n \in \mathbb{N}}\left(Z_{n}+\xi_{n}\right)
$$

again a (inhomogeneous) Boolean model.

Motivated by the preceding observations we can define a general Boolean model as $Z=\cup_{K \in X} K$, where $X$ is a Poisson particle process, i.e. a Poisson process on $\mathcal{C}^{d}$ with a locally finite intensity measure. Alternatively (and almost equivalently) we can start with a Poisson process $\Psi=\left\{\left(\xi_{n}, Z_{n}\right): n \in \mathbb{N}\right\}$ on $\mathbb{R}^{d} \times \mathcal{C}^{d}$, whose intensity measure $\tilde{\Lambda}$ satisfies (5), and define a Boolean model by (64). We take the second approach. The capacity functional of a general Boolean model $Z$ is given by the formula

$$
T_{Z}(C)=1-\exp \left\{-\int_{\mathbb{R}^{d} \times \mathcal{C}^{d}} \mathbf{1}\{(K+y) \cap C \neq \emptyset\} \tilde{\Lambda}(d(y, K))\right\} .
$$

Therefore the distribution of $Z$ determines the intensity measure (restricted to $\mathcal{C}^{d} \backslash\{\emptyset\}$ ) and hence the distribution of the associated Poisson particle process $X=\left\{Z_{n}+\xi_{n}: n \in\right.$ $\mathbb{N}\}$ (assuming that $X$ has a diffuse intensity measure). However it does not determine the intensity measure of $\Psi$. For an instationary random closed set $Z$, the volume fraction is defined as a function of $x \in \mathbb{R}^{d}$ by

$$
p(x):=\mathbb{P}(x \in Z)=T_{Z}(\{x\}) .
$$

Spezializing 65], we thus obtain for a Boolean model $Z$ that

$$
p(x)=1-\exp \{-\tilde{\Lambda}(\{(y, K): x \in K+y\})\}, \quad x \in \mathbb{R}^{d} .
$$

Let us fix a Boolean model as defined by (64) in terms of a Poisson process $\Psi=$ $\left\{\left(\xi_{n}, Z_{n}\right): n \in \mathbb{N}\right\}$ having intensity measure $\tilde{\Lambda}$. We assume that the measure $\Lambda:=$ $\tilde{\Lambda}\left(\cdot \times \mathcal{C}^{d}\right)$ is locally finite and diffuse. Then $\Phi:=\left\{\xi_{n}: n \in \mathbb{N}\right\}$ is a Poisson process with intensity measure $\Lambda$. Given $\Phi$, the grains $Z_{1}, Z_{2}, \ldots$ are conditionally independent, the conditional distribution being described by a stochastic kernel $\kappa$ from $\mathbb{R}^{d}$ to $\mathcal{C}^{d}$. This kernel is defined by

$$
\tilde{\Lambda}=\int_{\mathbb{R}^{d}} \int_{\mathcal{C}^{d}} \mathbf{1}\{(x, K) \in \cdot\} \kappa(x, d K) \Lambda(d x) .
$$

Given that $x$ is a point of $\Phi$, the probability measure $\kappa(x, \cdot)$ is the conditional distribution of the grain associated with $x$. The marked point process is an independent marking of $\Phi$ if and only if there is some probability measure $\mathbb{Q}$ (the mark distribution) such that $\kappa(x, \cdot)=\mathbb{Q}$ for $\Lambda$-a.e. $x \in \mathbb{R}^{d}$. In this case we call $Z$ a (inhomogeneous) Boolean model with independent grains. This definition depends not only on the distribution of $Z$, but also on the distribution of $\Psi$. It is possible that $Z$ can be represented in terms of two Poisson processes, where the first has independent marks and the second not. (In a sense this is the rule rather than the exception.)

Later (in Sect. 10.5) we will assume that

$$
\tilde{\Lambda}=\int_{\mathbb{R}^{d}} \int_{\mathcal{K}^{d}} \mathbf{1}\{(x, K) \in \cdot\} \gamma(x, K) d x \mathbb{Q}(d K),
$$


for some (measurable) function $\gamma$ on $\mathbb{R}^{d} \times \mathcal{K}^{d}$ and a probability measure $\mathbb{Q}$ on $\mathcal{K}^{d}$. This happens if and only if $\kappa(x, \cdot)$ is for $\Lambda$-a.e. $x \in \mathbb{R}^{d}$ absolutely continuous with respect to $\mathbb{Q}$. Then $\Psi$ has independent marks if and only if $\gamma(x, K)$ is independent of $K$ for $\mathbb{Q}$-a.e. $K$ and $\Lambda$-a.e. $x \in \mathbb{R}^{d}$. In this case $\gamma$ is the intensity function of the Poisson process $\Phi$.

\subsection{Instationary Cluster Models}

In this subsection we describe the direction dependent contact distribution function of an instationary Poisson cluster model. Let $\Phi_{p}$ be a Poisson process in $\mathbb{R}^{d}$ with absolutely continuous intensity measure as in (62) and intensity function $\gamma_{p}$. Let $\left\{N_{x}: x \in \Phi_{p}\right\}$ be a family of point processes that are conditionally independent given $\Phi_{p}$ and such that the conditional distribution of $N_{x}$ is the same for all $x \in \Phi_{p}$. As in the stationary case we may then introduce a typical cluster $N_{0}$ which satisfies $\mathbb{E} N_{0}\left(\mathbb{R}^{d}\right)<\infty$ and for which the reduced second moment measure of $N_{0}$ is absolutely continuous (compare Sect. 8.1]. Then we define the cluster process $\Phi$ as in (21) and impose the assumption that $\mathbb{E} \Phi(C)<\infty$ for all $C \in \mathcal{C}^{d}$. It is easy to see that

$$
\mathbb{E} \Phi(A)=\int_{A} \int_{\mathbb{R}^{d}} \gamma_{p}(x-y) \mathbb{E} N_{0}(d y) d x,
$$

for Borel sets $A \subset \mathbb{R}^{d}$, hence the intensity measure of $\Phi$ is absolutely continuous. The Poisson cluster model $Z$ is now obtained from $\Phi$ by independent marking as in the beginning of Sect. 8 Here we impose the integrability assumption

$$
\int_{\mathbb{R}^{d}} \mathbb{P}\left(\left(Z_{0}+x\right) \cap C \neq \emptyset\right) \mathbb{E} \Phi(d x)<\infty
$$

for all $C \in \mathcal{C}^{d}$, which is just assumption (5). Similarly to the stationary situation, $Z$ can be interpreted as an inhomogeneous Boolean model with independent grains and a typical grain as in (37). (Again this interpretation can be justified by a comparison of capacity functionals.) From this we can deduce a formula for $H_{B}(x, r)$. In order to obtain a local result, we have to proceed in a different way and combine results from [14] and [15]. Using the probability measures $\Gamma(\psi, \cdot)$ introduced in Sect.8.2 we define

$$
\mu_{0}^{\prime}(\cdot):=\mathbb{E}\left[\int_{\mathbb{R}^{d}} \int_{\mathbb{R}^{d} \times \mathcal{F} d} \mathbf{1}\{(y, A) \in \cdot\} \Gamma\left(\left(N_{0}-y\right)^{(0)}, d A\right) N_{0}(d y)\right] .
$$

This is a finite measure on $\mathbb{R}^{d} \times \mathcal{F}^{d}$. Assuming (as in Sect. 8.4) that $B^{*}$ and the typical grain $Z_{0}$ are almost surely in general relative position, we obtain

$$
\begin{aligned}
H_{B}(x, r, C)=\sum_{j=0}^{d-1}\left(\begin{array}{c}
d-1 \\
j
\end{array}\right) \int_{0}^{r} \int_{\mathbb{R}^{d} \times C} \int_{\mathbb{R}^{d} \times \mathcal{F}^{d}}\left(1-H_{B}(x, t)\right) t^{d-1-j} \\
\times \mathbf{1}\left\{d_{B}(-z-t u, A)>t\right\} \gamma_{p}(x+z+t u-y) \\
\times \mu_{0}^{\prime}(d(y, A)) \bar{\Theta}_{j}^{*}(B ; d(z, u)) d t .
\end{aligned}
$$

This result encompasses all results concerning Poisson cluster models or processes which we mentioned so far. It is a special case of Proposition 4.27 in [14] except that we do not have to assume that $B$ is smooth (see Theorem 5.1 in [15]). 


\subsection{Direction Dependent Contact Distributions of the General Boolean Model with Convex Grains}

We consider a general Boolean model $Z$ as defined in Sect.10.3. We assume that the underlying Poisson process $\Psi$ is concentrated on $\mathbb{R}^{d} \times \mathcal{K}^{d}$ and that it has an intensity measure $\tilde{\Lambda}$ of the form (66). Finally, we fix a gauge body $B$ and assume (as previously) that $B^{*}$ and the typical grain $Z_{0}$ are almost surely in general relative position. Then

$$
\begin{aligned}
H_{B}(x, r, C)=\sum_{j=0}^{d-1} & \left(\begin{array}{c}
d-1 \\
j
\end{array}\right) \int_{0}^{r} \int_{\mathcal{K}^{d}} \int_{\mathbb{R}^{d} \times C}\left(1-H_{B}(x, t)\right) t^{d-1-j} \\
& \times \gamma(x+z+t u, K) \Theta_{j}\left(K^{*} ; B ; d(z, u)\right) \mathbb{Q}(d K) d t,
\end{aligned}
$$

a result which has been proved in [15]. In the case of a Boolean model with independent grains the function $\gamma(x, K) \equiv \gamma(x)$ does not depend on $K$ and we obtain

$$
\begin{aligned}
H_{B}(x, r, C)=\sum_{j=0}^{d-1} & \left(\begin{array}{c}
d-1 \\
j
\end{array}\right) \int_{0}^{r} \int_{\mathbb{R}^{d} \times C}\left(1-H_{B}(x, t)\right) t^{d-1-j} \\
& \times \gamma(x+z+t u) \bar{\Theta}_{j}^{*}(B ; d(z, u)) d t
\end{aligned}
$$

Clearly, this formula is also a special case of [67), obtained by putting $N_{0}=\{0\}$. Equation 63 is recovered if $Z_{i}=\{0\}$ and $N_{0}=\{0\} \mathbb{P}$-a.s.

For a Boolean model with independent grains we now explore the kind of information about $(\gamma, \mathbb{Q})$ which we can retrieve from knowledge of the contact distribution functions of $Z$. Under suitable continuity and integrability assumptions we obtain from 69] that

$$
\frac{H_{B}^{\prime}(x, t, C)}{1-H_{B}(x, t)}=\sum_{j=0}^{d-1}\left(\begin{array}{c}
d-1 \\
j
\end{array}\right) t^{d-1-j} \int_{\mathbb{R}^{d} \times C} \gamma(x+z+t u) \bar{\Theta}_{j}^{*}(B ; d(z, u)),
$$

where the derivative of $H_{B}(x, t, C)$ is taken with respect to $t$. This already indicates that in its present form, the contact distribution functions may determine certain mean values for any choice of a measurable set $C \subset \mathbb{R}^{d}$ and a strictly convex body $B \in \mathcal{K}^{d}$. However, in order to make significant further progress and to determine spatial densities or distributions, it seems to be necessary to extend the notion of a contact distribution function by including additional second order information. This subject will be pursued more thoroughly in the next section.

\subsection{A Boolean Model with Spherical Grains}

The following special but important example shows the sort of information we can hope to extract from the contact distribution in its present form. Let $Z$ be an inhomogeneous Boolean model with independent grains. We assume that the typical grain is of the (randomly scaled) form $\tau B^{d}$, where $\tau$ has finite moments up to order $d-1$. We also assume that the intensity measure of the germ process has a continuous and bounded density $\gamma(x)$. In this special situation, we can deduce from 70 that the local contact 
distribution functions $H_{B^{d}}(x, t, C)$ (actually the local hazard rates), for $x \in \mathbb{R}^{d}, t \geq 0$ and measurable sets $C \subset S^{d-1}$, determine

$$
\sum_{j=0}^{d-1}\left(\begin{array}{c}
d-1 \\
j
\end{array}\right) t^{d-1-j} \mathbb{E}\left[\tau^{j} \gamma(x-\tau u-t u)\right]
$$

for all $x \in \mathbb{R}^{d}, t \geq 0$ and $u \in S^{d-1}$. Setting $t=0$, we find that $\mathbb{E}\left[\tau^{d-1} \gamma(x-\tau u)\right]$ is determined for all $x \in \mathbb{R}^{d}$ and $u \in S^{d-1}$. Replacing in this expression $x$ by $x-t u$, we find that $\mathbb{E}\left[\tau^{d-1} \gamma(x-\tau u-t u)\right]$ is determined as well. Hence we can conclude that

$$
\sum_{j=0}^{d-2}\left(\begin{array}{c}
d-1 \\
j
\end{array}\right) t^{d-2-j} \mathbb{E}\left[\tau^{j} \gamma(x-\tau u-t u)\right]
$$

is also determined for all $x \in \mathbb{R}^{d}, t \geq 0$ and $u \in S^{d-1}$. This argument can be repeated until we finally get that $\mathbb{E}[\gamma(x-\tau u)]$ is determined for all $x \in \mathbb{R}^{d}$ and $u \in S^{d-1}$. Thus we obtain at least some information on $\gamma$ of convolution type. Assuming additionally that $\gamma$ is constant along all lines of a known direction $u_{0} \in S^{d-1}$, we can infer that $\gamma$ and the moments $\mathbb{E} \tau^{j}, j=1, \ldots, d-1$, are determined. Another simple case where $\gamma$ is determined arises if $\tau$ is deterministic and known. We remark that nonspherical convex randomly scaled grains can be treated similarly, if an appropriate gauge body $B$ is chosen instead of the unit ball $B^{d}$. In general, however, we cannot expect to determine $\gamma$ or the distribution of $\tau$, on the basis of the information which is encoded in the kind of contact distribution functions we have considered up to now.

\section{Smooth Boolean Models and Second Order Information}

\subsection{Smooth Boolean Models}

The example at the end of the preceding section shows that even for a very special Boolean model $Z$, the contact distribution functions studied so far do not contain enough information to determine all characteristic quantities of $Z$. Therefore we extend the notion of a contact distribution again by including additional, second order information about $Z$ in its definition. At the same time, we will restrict our attention to certain classes of Boolean models which are still sufficiently general to describe many situations which are considered in practice.

For the following considerations, we concentrate on (non-stationary) Boolean models $Z$ with independent convex grains which fulfill certain smoothness conditions. We assume that the intensity measure $\tilde{\Lambda}$ of the underlying Poisson process $\Psi$ has the form

$$
\tilde{\Lambda}=\int_{\mathcal{K}^{d}} \int_{\mathbb{R}^{d}} \mathbf{1}\{(x, K) \in \cdot\} \gamma(x) d x \mathbb{Q}(d K),
$$

with the spatial intensity function $\gamma$ and the distribution $\mathbb{Q}$ of the typical grain. In addition, we assume that

(i) $\gamma$ is bounded and continuous,

(ii) $K$ is of class $C_{+}^{2}$ for $\mathbb{Q}$-a.e. $K \in \mathcal{K}^{d}$.

We then call $Z$ a smooth Boolean model. 


\subsection{Contact Distributions with Local Information}

Let $Z$ be a smooth Boolean model and $x \in \mathbb{R}^{d}$. For almost all realizations of $Z$ such that $x \notin Z$ there is a unique boundary point (contact point) $y \in Z$ such that $d(x, Z)=d(x, y)$ and $y$ lies in the boundary of a unique particle $K(x, Z)=L+z,(z, L) \in \Psi$. Note that $L+z$ has outer normal $-u(x, Z)$ at $y$. Then we define

$$
\tilde{s}(x, Z):=\left(\tilde{s}_{1}(x, Z), \ldots, \tilde{s}_{d-1}(x, Z)\right) \in \mathbb{R}^{d-1},
$$

where $\tilde{s}_{j}(x, Z):=s_{j}(K(x, Z),-u(x, Z))$ is the $j$-th normalized elementary symmetric function of the principal radii of curvature of the convex body $K(x, Z)$ in direction $-u(x, Z)$. The (extended) generalized contact distribution function of $Z$ is now given as

$$
H(x, r, C \times D):=\mathbb{P}(d(x, Z) \leq r, u(x, Z) \in C, \tilde{s}(x, Z) \in D \mid x \notin Z),
$$

for all $x \in \mathbb{R}^{d}, r \geq 0$ and measurable sets $C \subset S^{d-1}, D \subset \mathbb{R}^{d-1}$. Extended generalized contact distributions of a similar kind have been introduced and studied for much more general random closed sets $Z$; see [15].

Knowing all the conditional probabilities in 73 is equivalent to knowing the conditional expectations

$$
\mathbb{E}[\mathbf{1}\{d(x, Z) \leq r\} h(u(x, Z), \tilde{s}(x, Z)) \mid x \notin Z]
$$

for all $x \in \mathbb{R}^{d}$ and all measurable functions $h: S^{d-1} \times \mathbb{R}^{d-1} \rightarrow[0, \infty)$. In addition to distributional information about the position of the contact point in $Z$ realizing the distance from $x$ to $Z$, these conditional probabilities and expectations also contain second order information about $Z$ in a (arbitrarily small) neighbourhood of this contact point (local information). In the remaining part of this section, we describe the extent to which $\gamma$ and $\mathbb{Q}$ are determined by the contact distribution functions of the form $(73)$, i.e. we study the sort of information about the characteristics of $X$ which is implicitly included in (73).

The crucial relationship which we use for our analysis is

$$
\begin{gathered}
\mathbb{E}[\mathbf{1}\{d(x, Z) \leq r\} h(u(x, Z), \tilde{s}(x, Z)) \mid x \notin Z] \\
=\sum_{j=0}^{d-1}\left(\begin{array}{c}
d-1 \\
j
\end{array}\right) \int_{0}^{r} t^{d-1-j}(1-H(x, t)) \int_{\mathcal{K}^{d}} \int_{S^{d-1}} h(-u, s(K, u)) \\
\quad \times \gamma(x-t u-\tau(K, u)) s_{j}(K, u) \mathcal{H}^{d-1}(d u) \mathbb{Q}(d K) d t,
\end{gathered}
$$

where $s(K, u):=\left(s_{1}(K, u), \ldots, s_{d-1}(K, u)\right) \in \mathbb{R}^{d-1}$. More general formulas, relating conditional expectations of locally defined functionals of $Z$ to mean values of $\gamma$ (of convolution type) with respect to $\mathbb{Q}$, have been established in [15]. For instance, as in the preceding sections one can consider other gauge bodies than the unit ball, drop the smoothness assumptions on $(\gamma, \mathbb{Q})$, use local functionals of $Z$ different from $\tilde{s}(x, Z)$, and one can even avoid the Poisson assumption on $\Psi$. Relation (74) implies that the contact distribution functions $(73)$ determine the mean values 


$$
\int_{\mathcal{K}^{d}} \gamma(x-\tau(K, u)) g(s(K, u)) \mathbb{Q}(d K),
$$

for all $x \in \mathbb{R}^{d}, u \in S^{d-1}$ and all measurable functions $g: \mathbb{R}^{d-1} \rightarrow[0, \infty)$. Subsequently, we consider the difficult inverse problem of retrieving information about $(\gamma, \mathbb{Q})$ from these mean values.

\subsection{Independent Marking with Respect to Tangent Points}

A situation of a very special nature is given if there is a direction $u_{0} \in S^{d-1}$ such that the mark distribution $\mathbb{Q}$ of $\Psi$ is concentrated on the set $\left\{K \in \mathcal{K}^{d}: \tau\left(K, u_{0}\right)=0\right\}$. This situation corresponds to the choice of the center map $c(K)=\tau\left(K, u_{0}\right), K \in \mathcal{K}^{d}$, (compare Sect. 3.3); hence, $c(K)$ is the unique 'tangent point' of $K$ with exterior unit normal $u_{0}$. Note that $c(K)$ is uniquely defined for $\mathbb{Q}$-a.e. $K \in \mathcal{K}^{d}$. Then, for the choice $u=u_{0}$, the expression in (75) simplifies to

$$
\gamma(x) \int_{\mathcal{K}^{d}} g\left(s\left(K, u_{0}\right)\right) \mathbb{Q}(d K),
$$

for all $x \in \mathbb{R}^{d}$ and measurable functions $g: \mathbb{R}^{d-1} \rightarrow[0, \infty)$, since $\tau\left(K, u_{0}\right)=0$ for $\mathbb{Q}$-almost all $K \in \mathcal{K}^{d}$. Therefore the spatial density $\gamma$ and the distribution $\mathbb{Q}(\{K$ : $\left.\left.s\left(K, u_{0}\right) \in \cdot\right\}\right)$ are uniquely determined by (73). A particular case arises if $Z$ is stationary, since then we may choose $c(K)=\tau(K, u)$, for any $u \in S^{d-1}$. In this case, we thus find that the intensity $\gamma$ of $X$ and the distribution of the function $s\left(Z_{0}, u\right)$ of the typical grain $Z_{0}$ of $\Psi$ are determined by $(73)$. This result then holds for any other center map as well since the intensity $\gamma$ remains the same and the grain distribution given for the new center map is a well-defined image of $\mathbb{Q}$. (Note that $s_{j}(K, u)=s_{j}(K+x, u$ ) for all $K \in \mathcal{K}^{d}$ of class $C_{+}^{2}, x \in \mathbb{R}^{d}$ and $u \in S^{d-1}$.)

Note that for a stationary Boolean model, it is known that the point process of tangent points determines all parameters of the Boolean model (see [28, 30]). However, this point process is not used directly in the preceding investigation.

\subsection{The Case of Integrable Intensity Functions}

We return to a general smooth Boolean model, but assume now that $\gamma$ is integrable. Then, integrating with respect to $x \in \mathbb{R}^{d}$, using Fubini's theorem and exploiting the translation invariance of Lebesgue measure, we see that (75) implies that the distribution

$$
\mathbb{Q}(\{K: s(K, u) \in \cdot\})
$$

is determined for all $u \in S^{d-1}$. As a simple consequence we obtain that the mean surface measures $\bar{S}_{j}$ of the particles are determined by (73), since

$$
\int_{\mathcal{K}^{d}} S_{j}(K, C) \mathbb{Q}(d K)=\int_{C} \int_{\mathcal{K}^{d}} s_{j}(K, u) \mathbb{Q}(d K) \mathcal{H}^{d-1}(d u),
$$

for all measurable sets $C \subset S^{d-1}$. The above argument can be extended to the class of intensity functions which are integrable on a subspace and enjoy a partial invariance property with respect to the orthogonal subspace; see [15] for a precise statement. 


\subsection{Homothetic Particles}

Another special case where we obtain further results is that of a smooth Boolean model (with independent grains) where the particles are multiples of certain fixed shapes and the spatial intensity function $\gamma$ is integrable.

In a first example of that kind, we consider a typical grain $Z_{0}$ of the form

$$
Z_{0}=\tau Z_{0}^{\prime},
$$

where $\tau>0$ is a random variable and $Z_{0}^{\prime}$ is a random convex body (of class $C_{+}^{2}$ ) with $Z_{0}^{\prime} \in\left\{K_{1}, \ldots, K_{m}\right\}$. In addition, we assume that there is some $i \in\{1, \ldots, m\}$ such that

$$
\mathbb{P}\left(Z_{0}^{\prime}=K_{i}\right)>0 \quad \text { and } \quad \mathbb{P}\left(\tau \leq \epsilon \mid Z_{0}^{\prime}=K_{i}\right)>0,
$$

for all $\epsilon>0$. This condition means that arbitrarily small scaled copies of a particular shape can be observed with positive probability. Under these assumptions, not only the distribution in (76), but also the spatial density $\gamma$ is uniquely determined by (73).

A second example is given by (77) with $Z_{0}^{\prime} \in\left\{K_{1}, K_{2}\right\}$, where $K_{1}, K_{2}$ are two convex bodies that cannot be transformed into each other by translations and dilatations. Set $\alpha_{i}:=\mathbb{P}\left(Z_{0}^{\prime}=K_{i}\right), i \in\{1,2\}$, and assume that

$$
\limsup _{n \rightarrow \infty} \mathbb{E}\left[\tau^{n} \mid Z_{0}^{\prime}=K_{i}\right]^{\frac{1}{n}} / n<\infty
$$

whenever $\alpha_{i}>0$. Then, (73) determines $\alpha_{1}, \alpha_{2}$ and $\mathbb{P}\left(\tau \in \cdot \mid Z_{0}^{\prime}=K_{i}\right)$ whenever $\alpha_{i}>0$. Moreover, if the bodies $K_{1}, K_{2}$ are known, then $\mathbb{Q}=\mathbb{P}\left(Z_{0} \in \cdot\right)$ is determined as well. Under the additional assumption (78), $\gamma$ is also determined (compare the first example). Here, for $\alpha_{i}>0$, the weak growth condition (79) ensures that the conditional distribution $\mathbb{P}\left(\tau \in \cdot \mid Z_{0}^{\prime}=K_{i}\right)$ is determined by its moments $\mathbb{E}\left[\tau^{n} \mid Z_{0}^{\prime}=K_{i}\right]$, $n \in \mathbb{N}$.

In our final example, we consider a single shape, i.e. we assume that $Z_{0}=\tau K_{0}$, where $K_{0}$ is a fixed (but in general unknown) convex body and $\tau$ is normalized in the sense that $\mathbb{E} \tau=1$. Then $\gamma, \mathbb{Q}$ and $K_{0}$ are determined by the generalized contact distribution functions of $Z$ of the form (73). In order to illustrate the sort of information which is required on the right side of (73), let us consider the special case where we already know that $K_{0}=B^{d}$ is the Euclidean ball (compare the example at the end of Sect. 10). Hence $Z$ is a Boolean model with spherical grains. Then, for a point $x \notin Z$ we have to determine the direction $u(x, Z)$ of the unit vector pointing from $x$ to the nearest boundary point in $Z$, which is uniquely determined $\mathbb{P}$-a.s. Further, in this nearest point we have to measure the reciprocals of the principal curvatures of $\partial Z$ in order to get the value of $\tilde{s}_{1}(x, Z)$ (say); in the case of spherical grains, this value is equal to the radius of the $\mathbb{P}$-a.s. unique spherical particle of $Z$ nearest to $x$. Assuming that we can perform all these measurements for almost all points $x \notin Z$, we find that the spatial density $\gamma$ and the radial distribution of the spherical grains are determined by these measurements.

We remark that the above examples are special cases of more general results in [15]. 


\section{Final Remarks}

In this section we discuss some further extensions (and modifications) of contact distributions and indicate potential topics of future research. Throughout we fix a stationary random closed set $Z \subset \mathbb{R}^{d}$.

\subsection{General Sampling Schemes}

Let $B \in \mathcal{K}^{d}, 0 \in B$, be a gauge body. The contact distribution function $H_{B}$ can be interpreted as the distribution function of the relative distance of a "randomly chosen" point in the complement $\mathbb{R}^{d} \backslash Z$ of $Z$ from $Z$. It was proposed in [34] (see also [36]) to generalize this sampling scheme by choosing the reference point as the "typical point" of a random measure $\zeta$ on $\mathbb{R}^{d}$. To give a rigorous definition we assume that $\zeta$ has a finite and positive intensity $\mathbb{E} \zeta\left([0,1]^{d}\right)$ and that $\zeta$ is stationary jointly with $Z$. (The joint distribution of $Z$ and $\zeta$ is invariant under translations.) Let $\mathbb{P}_{\zeta}^{0}$ denote the Palm probability measure of $\zeta$ defined on the underlying sample space $(\Omega, \mathcal{A})$. The probability $\mathbb{P}_{\zeta}^{0}(A)$ of an event $A \in \mathcal{A}$ can be interpreted as the conditional probability of $A$ given that 0 is a typical point of $\zeta$ (see [22] for more formal definitions and further references). We then define

$$
H_{B, \zeta}(r):=\mathbb{P}_{\zeta}^{0}\left(d_{B}(0, Z \backslash\{0\}) \leq r\right), \quad r \geq 0 .
$$

Choosing $\zeta$ as the Lebesgue measure restricted to the complement of $Z$, we obtain the classical contact distribution function $H_{B}$ discussed in this paper. But there are many other interesting special cases. If, for instance, $Z=\zeta$ is a point process, then $H_{B^{d}, \zeta}$ is the nearest-neighbour distance distribution function of $Z$. As another example we might choose $\zeta$ as the $(d-1)$-dimensional Hausdorff-measure restricted to the exoskeleton $\operatorname{exo}_{B}(Z)$ of $Z$. (This requires suitable regularity properties of $\operatorname{exo}_{B}(Z)$.)

Definition 80 can be further extended by allowing random gauge bodies. A convenient way to accomplish this extension is to use a random field $Y=\left\{Y(x): x \in \mathbb{R}^{d}\right\}$ taking values in the set of all gauge bodies and being stationary jointly with $Z$ and $\zeta$. We may then define

$$
H_{Y, \zeta}(r):=\mathbb{P}_{\zeta}^{0}\left(d_{Y(0)}(0, Z \backslash\{0\}) \leq r\right), \quad r \geq 0 .
$$

For instance we might assume that the random gauge body $Y(0)$ is under $\mathbb{P}_{\zeta}^{0}$ independent of $Z$. Then $H_{Y, \zeta}$ is just a mixture of the distribution functions $H_{B, \zeta}$. A natural choice in such a setting (discussed in [34]) is $Y(0)=[0, U]$ (or $Y(0)=[-U, U]$ ), where $U$ is a random element of $S^{d-1}$. Another special case is to take $\zeta$ as the $(d-1)$ dimensional Hausdorff-measure restricted to the boundary of $Z$ and to define $Y(x)=$ $\left[0, \nu_{Z}(x)\right]$, where $\nu_{Z}(x)$ is the outer normal of $Z$ at the boundary point $x$. Then $H_{Y, \zeta}$ is the distribution of the distance of a typical boundary point $x$ of $Z$ from $Z \backslash\{x\}$ in outer normal direction. In this case $Y(0)$ and $Z$ are not independent under $\mathbb{P}_{\zeta}^{0}$.

\subsection{Intrinsic Volumes of Parallel Sets}

Up to affine transformation (see (9)) the contact distribution function $H_{B}(r)$ with respect to a gauge body $B$ is the volume fraction of the generalized parallel set 


$$
Z_{\oplus r}:=Z+r B^{*}
$$

Instead one can also study the means of other geometric functionals applied to (the suitably restricted) random set $Z_{\oplus r}$. An appropriate mathematical concept are the densities of additive functionals. Assume that $Z$ is a random $\mathcal{S}^{d}$-set, let $B$ be a strictly convex gauge body $B$ containing 0 in its interior, and let $W \in \mathcal{K}^{d}$ with $V_{d}(W)>0$. Under suitable integrability conditions the limit

$$
H_{B, j}(r, C)=\lim _{t \rightarrow \infty} \frac{\mathbb{E}\left[\Theta_{j}\left(Z_{\oplus r} \cap t W ; B^{*} ; \mathbb{R}^{d} \times C\right)\right]}{V_{d}(t W)}
$$

exists (and is finite) for any $j \in\{0, \ldots, d-1\}, r \geq 0$, and $C \subset \partial B^{*}$ (see [13] and [39]). Moreover, $H_{B, j}(r, \cdot)$ is a signed measure on $\partial B^{*}$. For $j=d-1$ this measure has been discussed in Sect. 9.2

The idea to study the behaviour of intrinsic volumes as a function of a thresholdparameter was first formulated in [27] in the context of continuous percolation. Further very fruitful applications of such and related ideas to the analysis of complex spatial structures occuring in statistical physics can be found in [25] and [26] (see also the contributions by H-J. Vogel (Chap. 1), by M. Lösche and P. Krüger (Chap. 2), by C. Beisbart et al. (Chap. 3), and by C. Beisbart, M. Kerscher and K. Mecke (Chap. 4), in this volume). In [16], the functions $H_{s, j}(r):=H_{B^{d}, j}\left(r, S^{d-1}\right)$ are used as summary statistics for the analysis of point processes $Z$ composed by the centers of galaxy clusters. Moreover, in [46] it is suggested to analyse means of various geometric functionals of eroded or dilated porous media in order to gain insight into their physical properties or for testing goodness-of-fit of potential models for such structures.

If $Z$ is a (stationary) Boolean model with typical convex grain $Z_{0}$, then $Z_{\oplus r}$ is a Boolean model with typical grain $Z_{0}+r B^{*}$. If $Z_{0}$ has an isotropic distribution it is possible to use general results for Boolean models (see e.g. [25, 39, 50]) to express the functions $H_{s, j}$ in terms of the intensity of the underlying Poisson process and the mean volumes $\mathbb{E} V_{j}\left(Z_{0}\right)$. In the non-isotropic case the formulas for $H_{s, 1}, \ldots, H_{s, d-2}$ become more complicated (see [50, 51]). We are not aware of any other (more or less) explicit analytic results.

\section{References}

1. Baddeley, A.J. (1999): 'Spatial sampling and censoring'. In: Stochastic Geometry: Likelihood and Computation, ed. by O. Barndorff-Nielsen, W. Kendall, M.N.M. van Lieshout (Chapman \& Hall/CRC, Boca Raton), pp. 37-78.

2. Baddeley, A.J., Gill, R.D. (1997): 'Kaplan-Meier estimators of distance distributions for spatial point processes'. Ann. Statist. 25, pp. 263-292.

3. Chiu, S.N., Stoyan, D. (1998): 'Estimators of distance distributions for spatial patterns'. Stat. Neerl. 52, pp. 239-246.

4. Daley, D.J., Vere-Jones, D. (1988): An Introduction to the Theory of Point Processes. (Springer, New York)

5. Hahn, U., Micheletti, A., Pohlink, R., Stoyan, D., Wendrock, H. (1999): 'Stereological analysis and modelling of gradient structures'. Journal of Microscopy 195, pp. 113-124. 
6. Hansen, M.B., Baddeley, A.J., Gill, R.D. (1996): 'Kaplan-Meier type estimators for linear contact distributions'. Scand. J. Statist. 23, pp. 129-155.

7. Hansen, M.B., Baddeley, A.J., Gill, R.D. (1999): 'First contact distributions for spatial patterns: regularity and estimation'. Adv. in Appl. Probab. 31, pp. 15-33.

8. Heinrich, L. (1992): 'On existence and mixing properties of germ-grain models'. Statistics 23, pp. 271-286.

9. Heinrich, L. (1993): 'Asymptotic properties of minimum contrast estimators for parameters of Boolean models'. Metrika 40, pp. 67-94.

10. Heinrich, L. (1998): 'Contact and chord length distribution of a stationary Voronoi tessellation'. Adv. in Appl. Probab. 30, pp. 603-618.

11. Hilfer, R. (2000): 'Local porosity theory and stochastic reconstruction for porous media'. In: Statistical Physics and Spatial Statistics, Lecture Notes in Physics 554, ed. by K. Mecke, D. Stoyan (Springer, Berlin), pp. 203-241.

12. Hug, D. (1999): Measures, curvatures and currents in convex geometry, Habilitationsschrift. (Albert-Ludwigs-Universität, Freiburg)

13. Hug, D. (2000): 'Contact distributions of Boolean models'. Rend. Circ. Mat. Palermo (2) Suppl. 65, pp. 137-181.

14. Hug, D., Last, G. (2000): 'On support measures in Minkowski spaces and contact distributions in stochastic geometry’. Ann. Probab. 28, pp. 796-850.

15. Hug, D., Last, G., Weil, W. (2002): 'Generalized contact distributions of inhomogeneous Boolean models'. Adv. in Appl. Probab. 34, pp. 21-47.

16. Kerscher, M. (2000): 'Statistical analysis of large-scale structure in the universe'. In: Statistical Physics and Spatial Statistics, Lecture Notes in Physics, ed. by K. Mecke, D. Stoyan (Springer, Berlin), pp. 36-71.

17. Kiderlen, M. (2001): 'Non-parametric estimation of the directional distribution of stationary line and fibre processes'. Adv. in Appl. Probab. 33, pp. 6-24.

18. Kiderlen, M., Weil, W. (1999): 'Measure-valued valuations and mixed curvature measures of convex bodies'. Geom. Dedicata 76, pp. 291-329.

19. Kingman, J. F. (1995): Poisson Processes (Oxford, Clarendon)

20. Last, G., Holtmann, M. (1999): 'On the empty space function of some germ-grain models'. Pattern Recognition 32, pp. 1587-1600.

21. Last, G., Schassberger, R. (1998): 'On the distribution of the spherical contact vector of stationary germ-grain models'. Adv. in Appl. Probab. 30, pp. 36-52.

22. Last, G., Schassberger, R. (2000): 'On stationary stochastic flows and Palm probabilities of surface processes'. Ann. Appl. Probab. 10, pp. 463-492.

23. Last, G., Schassberger, R. (2001): 'On the second derivative of the spherical contact distribution function of smooth grain models'. Probab. Theory and Related Fields 121, pp. 49-72.

24. Matheron, G. (1975): Random Sets and Integral Geometry. (Wiley, New York)

25. Mecke, K. (1994): Integralgeometrie in der Statistischen Physik. (Verlag Harri Deutsch, Frankfurt am Main)

26. Mecke, K. (2000): 'Additivity, convexity and beyond: applications of Minkowski functionals in statistical physics'. In: Statistical Physics and Spatial Statistics, Lecture Notes in Physics 554, ed. by K. Mecke, D. Stoyan (Springer, Berlin), pp. 112-184

27. Mecke, K., Wagner, H. (1991): 'Euler characteristics and related measures for random geometric sets'. J. Stat. Phys. 64, pp. 843-850.

28. Molchanov, I. (1995): 'Statistics of the Boolean model: from the estimation of means to the estimation of distributions'. Adv. in Appl. Probab. 27, pp. 63-86.

29. Molchanov, I. (1997): Statistics of the Boolean model for Practitioners and Mathematicians. (Wiley, Chichester) 
30. Molchanov, I., Stoyan, D. (1994): 'Asymptotic properties of estimators for parameters of the Boolean model'. Adv. in Appl. Probab. 26, pp. 301-323.

31. Muche, L., Stoyan, D. (1992): 'Contact and chord length distribution of the Poisson Voronoi tessellation'. J. Appl. Probab. 29, pp. 467-471.

32. Ohser, J., Mücklich, F. (2000): Statistical Analysis of Microstructures in Materials Science. (Wiley, Chichester)

33. Quintanilla, J., Torquato, S. (1997): 'Microstructure functionals for a model of statistically inhomogeneous random media'. Physical Review E 55, pp. 1558-1565.

34. Rataj, J., (1993): 'Random distances and edge correction'. Statistics 24, pp. 377-385.

35. Rataj, J., Saxl, I. (1995): 'Boolean cluster models: mean cluster dilations and spherical distances'. Mathematica Bohemica 122, pp. 21-36.

36. Saxl, I. (1993): 'Contact distances and random free paths'. Journal of Microscopy 170, pp. 53-64.

37. Schneider, R. (1993): Convex Bodies: the Brunn-Minkowski Theory, Encyclopedia of Mathematics and its Applications 44. (Cambridge University Press, Cambridge)

38. Schneider, R. (1994): 'An extension of the principal kinematic formula of integral geometry'. Rend. Circ. Mat. Palermo, II. Ser., 35, pp. 275-290.

39. Schneider, R., Weil, W. (2000): Stochastische Geometrie. (Teubner, Stuttgart)

40. Serra, J. (1982): Image Analysis and Mathematical Morphology. (Academic Press, London)

41. Soille, P. (1999): Morphological Image Analysis: Principles and Applications. (Springer, Berlin)

42. Stoyan, D., Kendall, W.S., Mecke, J. (1995): Stochastic Geometry and its Applications, Second Edition. (Wiley, Chichester)

43. Stoyan, D., Penttinen, A. (2000): 'Recent applications of point process methods in forestry statistics'. Statistical Science 15, pp. 61-78.

44. Stoyan, D., Stoyan, H. (1994): Fractals, Random Shapes and Point Fields: Methods of Geometrical Statistics. (Wiley, Chichester)

45. Stoyan, D., Stoyan, H., Tscheschel, A., Mattfeld, T. (2001): 'On the estimation of distance distribution functions for point processes and random sets'. Image Anal. Stereol. 20, pp. 65-69.

46. Tscheschel, A., Stoyan, D., Hilfer, R. (2000): 'Erosion-dilation analysis for experimental and synthetic microstructures of sedimentary rock'. Physica A 284, pp. 46-58.

47. Van Lieshout, M.N.M., Baddeley, A.J. (1995): 'A non-parametric measure of spatial interaction in point-patterns'. Statist. Neerland. 49, pp. 344-361.

48. Van Lieshout, M.N.M., Baddeley, A.J. (1999): 'Indices of dependence between types in multivariate point patterns'. Scand J. Statist. 26, pp. 511-532.

49. Weil, W. (1997): 'Mean bodies associated with random closed sets' Rend. Circ. Mat. Palermo (2) Suppl. 50, pp. 387-412.

50. Weil, W. (2000): 'Mixed measures and inhomogeneous Boolean models'. In: Statistical Physics and Spatial Statistics, Lecture Notes in Physics 554, ed. by K. Mecke, D. Stoyan (Springer, Berlin), pp. 95-110

51. Weil, W. (2001): 'Densities of mixed volumes for Boolean models' Adv. in Appl. Probab. 33, pp. 39-60.

52. Weil, W., Wieacker, J.A. (1988): 'A representation theorem for random sets'. Probab. Math. Statist. 9, pp. 147-151. 